\title{
Impact of Social Relation and Group Size in Multicast Ad Hoc Networks
}

\author{
Yi Qin, Riheng Jia, Jinbei Zhang, Weijie Wu, Xinbing Wang
}

\begin{abstract}
This paper investigates the multicast capacity of static wireless social networks. We adopt the two-layer network model, which includes the social layer and the networking layer. In the social layer, the social group size of each source node is modeled as power-law distribution. Moreover, the rank-based model is utilized to describe the relation between source and destinations in the networking layer. Based on the two-layer network model, the probability density function (PDF) of the destination positions is analyzed and verified by numerical simulation, which is different from the traditional ad hoc networks. According to the PDF, the bound of the network capacity is derived, and we propose a Euclidean minimum spanning tree based transmission scheme, which is proved to achieve the order of capacity bound for most cases. Finally, the capacity of social networks is compared with the traditional multicast ad hoc networks, which indicates that the capacity scaling performs better in social networks than traditional ones. To our best knowledge, this is the first work of analyzing the impact on the capacity of social relation and group size in multicast ad hoc networks for the rank-based model.
\end{abstract}

\section{INTRODUCTION}

Network scaling law was firstly studied by Gupta and Kumar [1], followed by many works about the network capacity and throughput [2]- [4]. However, their models fell short in well characterizing real social networks, and they had to consider simplified models instead. For example, in most of these works, the destinations associated with each user were drawn according to a uniform distribution, which is unrealistic. To give another example, it was also an unrealistic assumption that the number of friends was known apriori in traditional multicast ad hoc networks [2]- [4]. Therefore, many researchers turned to study social characteristics such as the way people selected friends (destinations) [5] [6] and the number of these friends [7] [8]. It was only recently that studying social phenomena had become a hot topic in ad hoc networks [9], peer to peer networks [10] and some other networks [11]- [14].

To study the social phenomenon, two characteristics of social networks are considered in our paper, which are the social group size and the social relation. Firstly, we introduce the group size which describes the number of friends for each node. In [7] [8] [15], the authors analyzed the probability that an arbitrary node had $q$ friends based on the data of Cyworld, MySpace and orkutwith, each with more than 10 million users. The results showed that the probability satisfied the power-law distribution, which generally matched the fact.

The authors are with the Department of Electronic Engineering Shanghai Jiao Tong University, China

Email: \{qinyi_33, jiariheng, abelchina,weijiewu, xwang8\}@sjtu.edu.cn
The second characteristic of social networks is the social relation which has been studied for more than thirty years [9], [16]- [19]. In 1979, the authors in [16] focused on about 500 persons in a network. Further research about 130 million persons of Myspace was demonstrated in [17]. In their works, the social network was modeled as a graph to describe the geographical and social relations among users. Moreover, the social relation in these papers reflected how users select friends in the network, and it was usually determined by the factors such as friendship, common interest or alliance, which was different from the traditional ad hoc networks. From the above studies of the experiments about how people selected friends, some feasible social relation models were proposed such as distance-based model and rank-based model [19]. The scaling laws of these two models were analyzed in [9] and [18], respectively. In [9], the probability that node $j$ was a friend of node $i$ was proportional to $d(i, j)^{-\alpha}$, where $d(i, j)$ represented the distance between $i$ and $j$. Instead, in [18], the authors illustrated that the probability was proportional to $\operatorname{Rank}_{i}^{-\alpha}(j)$, where $\operatorname{Rank}_{i}(j)$ was the rank of $j$ with respect to $i$.

The two social characteristics above were both observed through statistics from online social networks. However, they can also be introduced into wireless networks because that they are independent from the wireless nature of the networks. Moreover, it is worthwhile to study wireless social networks due to the two facts: 1) The study of wireless social networks is promising for the reason that the social wireless networks, such as MSN wireless client, facebook wireless client, have gained a large popularity nowadays. Furthermore, in cellular networks, as the number of wireless terminals increases, one basestation needs to service more and more terminals. However, the number of simultaneously serviced terminals is restricted due to the limited up-link resource. This will reduce the per-terminal throughput when there are too many terminals. Therefore, wireless multi-hop transmission can be adopted to improve the per-terminal throughput of social networks. 2) The multi-hop user relaying technology has become an important topic and been well studied. It can be demonstrated from [20], which investigated coordination among users through wireless channel (such as multi-hop), that the user relaying technology helped to improve the capacity of wireless system.

Additionally, the nodes in the network need to transmit their packets to all of their friends by multicasting in this paper, which is also a common requirement. For example, a user of facebook may want to send a message to all of his friends.

The capacity of traditional multicast ad hoc networks was intensively studied in [2]- [4]. However, the traditional results cannot be directly applied to social networks, and therefore 
we present this paper to improve and generalize the theory of ad hoc network capacity. In particular, the main difference between the social networks and the traditional ad hoc networks is the Probability Distribution Function (PDF) of destination positions. Therefore, a challenging question arises when considering the two social characteristics:

- How do the two characteristics of static social networks jointly impact the capacity of network, and what is the capacity achieving scheme?

To answer this question, we study the two-layer static social network model, which includes social layer and networking layer. In the social layer, the social group size of each source node was modeled as power-law distribution model. Moreover, the rank-based model was utilized to describe the relation between source and destinations in the networking layer. For a node with $q$ friends, the PDF of its friends' positions are derived in our paper. The simulations of the PDF are also illustrated, which verify the theoretical results. We then analyze the bound of network capacity and propose a capacity achieving scheme based on the Euclidean Minimum Spanning Tree.

The main contributions of this paper are summarized as follows:

- The relation between rank and geographical position of an arbitrary node is demonstrated for rank-based static social network model. If there are $n$ nodes in the network which is of size $1 \times 1$, the result shows that $x=\Theta\left(\sqrt{\frac{\operatorname{Rank}}{n}}\right) 1$ with probability 1 when $n$ goes to infinity, where $x$ is the distance between source and destination. Moreover, we also derive the PDF of the destination positions for a given source, which is verified by the simulations.

- We analyze the capacity bound of the social network model based on the PDF of destination positions and Euclidean Minimum Spanning Tree. Moreover, a corresponding transmission scheme is proposed to achieve the capacity bound in most cases.

- Finally, we compare the capacity of social networks with the traditional ad hoc networks, and the results indicate that the capacity scaling performs better in social networks than traditional ad hoc networks.

The rest of this paper is organized as follows. In Section II, we introduce the network model and definitions. The PDF of destination positions and the capacity bound of the social network model are derived in Section III, and the theoretical results of the PDF is verified by numerical simulations in Section IV. In Section V, a capacity achieving scheme is proposed to demonstrate that the capacity is achievable in most cases. In Section VI, we compare the capacity of social networks with traditional ad hoc networks. Finally, we conclude in Section VII.

\footnotetext{
${ }^{1}$ We use standard asymptotic notations in our paper. Consider two nonnegative function $f(\cdot)$ and $g(\cdot)$ : (1) $f(n)=o(g(n))$ means $\lim _{n \rightarrow \infty} f(n) / g(n)=0$. (2) $f(n)=O(g(n))$ means $\lim _{n \rightarrow \infty} f(n) / g(n)<\infty$. (3) $f(n)=\omega(g(n))$ means $\lim _{n \rightarrow \infty} f(n) / g(n)=\infty$. (4) $f(n)=\Omega(g(n))$ means $\lim _{n \rightarrow \infty} f(n) / g(n)>0$. (5) $f(n)=\Theta(g(n))$ means $f(n)=O(g(n))$ and $g(n)=O(f(n))$. (6) $f(n)=\tilde{\Theta}(g(n))$ means that there exists two constants $a$ and $b$ satisfy $f(n)=O\left(g(n) \log ^{a} n\right)$ and $f(n)=\Omega\left(g(n) \log ^{b} n\right)$
}

\section{Network Model And Definitions}

In this section, the two-layer model of static social networks is proposed based on group size and social relation, which are the main differences between social network model and traditional ad hoc network model. Moreover, we also list some network performance metrics and notations for our following analysis.

\section{A. The Social Network Model}

In this paper, we adopt the two-layer network model, which includes

1) Social layer: This layer captures the social relation among individuals, which is not related with the network topology.

2) Networking layer: This layer reflects the network topology based on the node positions.

In the social layer, each node $i$ selects some friends as its destinations, which form a social group. Moreover, the size of the social group, denoted as $q_{i}$, is proved to satisfy the powerlaw degree distribution in [9]. In particular, the experiments of three online social networking services, each with more than 10 million users, indicate that $\mathbb{P}\left\{q_{i}=q\right\}$ follows the distribution in (1)

$$
\mathbb{P}\left\{q_{i}=q\right\}=\frac{1}{G_{n} q^{\beta}},
$$

where $\beta \geq 0$ is a constant and $G_{n}=\sum_{q=1}^{n} \frac{1}{q^{\beta}}$.

In the networking layer, we consider the network which is a unit square, and $n$ static nodes (users) are uniformly and randomly distributed in it. The well-known protocol model as in [1] is employed as the interference model in our network. When node $i$ wants to transmit a packet to node $j$, the transmission is considered to be successful if the following inequality is satisfied

$$
\left\|X_{i}-X_{j}\right\| \leq r(n)
$$

where $X_{i}$ represents node $i$ 's location, and $r(n)$ is the maximum transmission range of each node, which is a function of $n .\|\cdot\|$ here is the Euclidean distance. Moreover, any other node $k$ who wants to transmit packet at the same time must satisfy the inequality as

$$
\left\|X_{k}-X_{j}\right\| \geq(1+\Delta) r(n),
$$

where $\Delta>0$ is a constant factor depending on the acceptable Signal to Interference Noise Ratio (SINR) of the network. Furthermore, the bandwidth of the network is finite and constant. In this model, the transmission range $r(n)$ is assumed as $r(n)=\frac{\sqrt{\log n}}{\sqrt{n}}[1]$, which guarantees the connectivity of the network. In addition, each node transmits packet to its destinations through multi-hop.

It is important to study the node mapping from the social layer to the networking layer. We adopt the rank-based model in [18] and [19]. In this model, for a given node $i$, when it selects one friend from all the nodes, we denote that node $j$ is selected with probability $P_{i \rightarrow j}$. Based on social experiments [19], the friends of a user are more likely to be close to him. 
Denoting $P$ as the set consisting all the nodes and $d(i, j)=$ $\left\|X_{i}-X_{j}\right\|$ as the distance between $i$ and $j$, the rank of $j$ with respect to $i$ is defined as

$$
\operatorname{Rank}_{i}(j)=|\{k \in P: d(i, k)<d(i, j)\}|,
$$

where $|\cdot|$ represented the number of elements in the set. According to [18] and [19], $P_{i \rightarrow j}$ satisfies

$$
P_{i \rightarrow j} \propto \frac{1}{\operatorname{Rank}_{i}^{\alpha}(j)},
$$

where $\alpha \geq 0$ is a constant. When $\alpha=0$, the network is the traditional ad hoc network. (5) shows the fact that a node is more likely to select a friend that nearby. Furthermore, this model focuses on relative location instead of geographic location. We define $H_{n}=\sum_{i=1}^{n} \frac{1}{i^{\alpha}}$. Therefore, by normalizing, (5) can be represented as

$$
P_{i \rightarrow j}=\frac{1}{H_{n} \operatorname{Rank}_{i}^{\alpha}(j)} .
$$

In [19], the authors verify the (6) by online data. It should be noticed that we consider the case that one node selects multiple destinations instead of one. Therefore, the distribution of the destinations will be analyzed based on both social group size and (6).

\section{B. Network Performance Metrics and Notations}

We give the definitions of throughput and capacity in this subsection. Moreover, some notations are also demonstrated.

Definition of throughput: For a given scheme, we define the throughput as the maximum achievable transmission rate. In $t$ time slots, any $i$ 's destination (denoted as $k$ ) receives $M_{i}(k, t)$ packets and the number of $i$ 's friends is $q_{i}$. The long term per-node throughput of this multicast session is defined by $\lambda_{i}(n)$ as $\lambda_{i}(n)=\liminf _{t \rightarrow \infty} \frac{1}{t q_{i}} \sum_{k=1}^{q_{i}} M_{i}(k, t)$. Then the average throughput of this scheme is defined by $T(n)$

$$
T(n)=\liminf _{n \rightarrow \infty} \frac{1}{n} \sum_{i=1}^{n} \lambda_{i}(n) .
$$

Definition of capacity: In this social network, $C(n)$ is said to be the asymptotic per-node social multicast capacity with order $\Theta\left(T_{0}(n)\right)$, where $T_{0}(n)$ satisfies 8 .

$$
\begin{aligned}
& \left\{T(n): \lim _{n \rightarrow \infty} \frac{T(n)}{T_{0}(n)}=\infty\right\}=\emptyset, \\
& \left\{T(n): \lim _{n \rightarrow \infty} \frac{T(n)}{T_{0}(n)}>0\right\} \neq \emptyset,
\end{aligned}
$$

for any feasible $T(n)$.

Furthermore, we list some important parameters in Table I that will be used in later analysis, proofs and discussions.

\section{CAPACITy ANAlysis For Social Networks}

In this section, firstly, the relation between rank and geographical position of an arbitrary node is analyzed. Based on such relation, we derive the PDF of the destinations, and finally obtain the bound of capacity.

The links between the nodes belonging to one multicast session are considered, which can organize a spanning tree.
TABLE I: Important Definitions of Symbols and Notations

\begin{tabular}{|c|l|}
\hline Symbol & Definition \\
\hline$n$ & Number of users \\
\hline$r(n)$ & The maximum transmission range \\
\hline$q_{i}$ & Number of destinations (friends) of user $i$ \\
\hline$\alpha$ & Parameter of rank-based model \\
\hline$\beta$ & $\begin{array}{l}\text { Parameter of power-law distribution of } \\
\text { group size }\end{array}$ \\
\hline$R a n k_{i}(j)$ & The rank of $j$ with respect to $i$ \\
\hline$f_{n}(x, \theta)$ & $\begin{array}{l}\text { The probability density function of destina- } \\
\text { tion positions }\end{array}$ \\
\hline$\overline{\|E M S T\|}$ & The total length of the EMST \\
\hline$C(n)$ & The capacity of the network \\
\hline$N_{q}(n)$ & $\begin{array}{l}\text { The minimum total number of hops for a } \\
\text { multicast tree with } q+1 \text { nodes }\end{array}$ \\
\hline$R_{q}$ & $\begin{array}{l}\text { The capacity ratio of social networks and } \\
\text { traditional ad hoc networks when each node } \\
\text { has } q \text { friends }\end{array}$ \\
\hline
\end{tabular}

Thus, in order to minimize the length of transmission path, the Euclidean Minimum Spanning Tree (EMST) is investigated. The total length for this EMST is proved in [21] to satisfy the following lemma.

Lemma 1 Let $f(\mathbf{x})$ denote the PDF of the related nodes in the network, where $\mathrm{x}$ is the position vector. Then, for large number of nodes $n$ and the network dimension $d>1$, if $f(\mathbf{x})$ is independent from $n$, the total length for the EMST satisfies

$$
\lim _{n \rightarrow \infty} n^{-\frac{d-1}{d}} \overline{\|E M S T\|}=c(d) \int_{R^{d}} f(\mathbf{x})^{\frac{d-1}{d}} d \mathbf{x},
$$

with probability 1 , where $c(d)$ is constant.

The proof of this lemma is demonstrated in [21], and $d=2$ in our paper. However, when $f(\mathbf{x})$ is related with $n$, Lemma 1 does not hold. As a result, we denote the PDF as $f_{n}(\mathbf{x})$ in this paper and give the following lemma which indicates the bound of the total length of the EMST for infinite $n$.

Lemma 2 If $f_{n}(\mathbf{x})$ in Lemma 1 is related with infinite $n$ and the following two conditions are satisfied,

- Condition 1: There exists a function $g_{n}(\mathbf{x})=$ $\sum_{\xi_{i} \in \Psi} \gamma_{i} 1_{\xi_{i}}$ satisfying

$$
\int_{\Psi}\left|g_{n}(\mathbf{x})-f_{n}(\mathbf{x})\right| d \mathbf{x} \rightarrow 0
$$

when $n$ goes to infinity, where $\Psi$ is the range of total network, $\xi_{i}(i=1,2, \cdots)$ is the partition of $\Psi$ separating $\Psi$ into many non-overlapping parts (i.e., $\xi_{i} \cap \xi_{j}=\emptyset, \forall i \neq$ $j$ and $\left.\bigcup \xi_{i}=\Psi\right), 1_{\xi_{i}}$ is an indicative function and $\int_{\xi_{i}} g_{n}(\mathbf{x}) d \mathbf{x}=\int_{\xi_{i}} f_{n}(\mathbf{x}) d \mathbf{x}$. Moreover, each part $\xi_{i}$ is a $d$-dimensional hypercube, and the number of its adjacent parts is limited by a constant $\zeta$.

- Condition 2: $\lim _{n \rightarrow \infty} n \int_{\xi_{i}} g_{n}(\mathbf{x}) d \mathbf{x}=\Omega(1)$ with probability 1. 
One than has that with probability 1

$$
\lim _{n \rightarrow \infty} \frac{n^{\frac{-d-1}{d}} \overline{\|E M S T\|}}{\int_{R^{d}} f_{n}(\mathbf{x})^{\frac{d-1}{d}} d \mathbf{x}}=c(d),
$$

where $c(d)$ is constant.

Proof: The proof can be found in the Appendix.

Moreover, according to the sum of $p$-series, the $G_{n}$ in (1) and $H_{n}$ in 6 can be represented as

$$
H_{n}=\sum_{i=1}^{n} \frac{1}{i^{\alpha}}=\left\{\begin{array}{lc}
\Theta\left(n^{1-\alpha}\right) & 0 \leq \alpha<1, \\
\Theta(\log n) & \alpha=1 \\
\Theta(1) & \alpha>1,
\end{array}\right.
$$

and

$$
G_{n}=\sum_{q=1}^{n} \frac{1}{q^{\beta}}=\left\{\begin{array}{lc}
\Theta\left(n^{1-\beta}\right) & 0 \leq \beta<1, \\
\Theta(\log n) & \beta=1, \\
\Theta(1) & \beta>1 .
\end{array}\right.
$$

To calculate the PDF of user $i$ 's $q_{i}$ friends, the relation between rank and geographical position is important. We will show this relation in Lemma 4 which is supported by Lemma 3. It should be noticed that the impact of boundary effect on scaling laws can be ignored in the proofs of this paper, which can also be found in other works of scaling laws [2].

Lemma 3 We denote two constants $a$ and $b$ where $0<a<1$ and $b>1$. For any $X \in\{1,2, \cdots, n\}$, there must be

$$
\frac{n !}{X !(n-X) !}\left(\frac{a X}{n}\right)^{X}\left(1-\frac{a X}{n}\right)^{n-X}<c_{1}\left(a e^{1-a}\right)^{X},
$$

where $\mathrm{e}$ is the base of the natural logarithm and $c_{1}$ is constant, when $n$ is large enough. Moreover, if $b X \leq n$, there must be

$$
\frac{n !}{X !(n-X) !}\left(\frac{b X}{n}\right)^{X}\left(1-\frac{b X}{n}\right)^{n-X}<c_{2}\left(b e^{1-b}\right)^{X},
$$

where $c_{2}$ is constant and $n$ is large enough.

Proof: Firstly, the left-hand side of (14) satisfies

$$
\begin{aligned}
g(X) & =\frac{n !}{X !(n-X) !}\left(\frac{a X}{n}\right)^{X}\left(1-\frac{a X}{n}\right)^{n-X} \\
& \stackrel{(a)}{<} \frac{c_{3}\left(\frac{n}{e}\right)^{n} \sqrt{n}}{\left(\frac{X}{e}\right)^{X} \sqrt{X}\left(\frac{n-X}{e}\right)^{n-X} \sqrt{n-X}} \frac{a^{X} X^{X}}{n^{X}} \frac{(n-a X)^{n-X}}{n^{n-X}} \\
& =c_{3} a^{X}\left(\frac{n-a X}{n-X}\right)^{n-X} \sqrt{\frac{1}{X}+\frac{1}{n-X}} \\
& <2 c_{3} a^{X}\left(\frac{n-a X}{n-X}\right)^{n-X}
\end{aligned}
$$

where $c_{3}>1$ is constant, and $(a)$ holds due to the fact that

$$
\lim _{n \rightarrow \infty}\left(\frac{n}{e}\right)^{n} \frac{\sqrt{2 \pi n}}{n !}=1
$$

Moreover, for any $c>0$, there must be $1<(1+c)^{\frac{1}{c}}<e$. Hence, $g(X)$ satisfies

$g(X)<2 c_{3} a^{X}\left(\left(1+\frac{(1-a) X}{n-X}\right)^{\frac{n-X}{(1-a) X}}\right)^{(1-a) X}<2 c_{3}\left(a e^{1-a}\right)^{X}$

Therefore, if we define $c_{1}=2 c_{3}$, equation (14) is proved. Furthermore, equation (15) can be proved in the similar way.
Lemma 4 Node $\mathrm{i}$ is the source node. If the rank of node $\mathrm{j}$ to $\mathrm{i}$ is $\operatorname{Rank}_{i}(\mathrm{j})=X$, the distance between node $\mathrm{i}$ and $\mathrm{j}$ satisfies $x=\Theta\left(\sqrt{\frac{X}{n}}\right)$ with probability 1 , when $n \rightarrow \infty$.

Proof: The probability that the distance between node $i$ and $j$ does not follow $x=\Theta\left(\sqrt{\frac{X}{n}}\right)$ is

$1-\mathbb{P}\left(x=\Theta\left(\sqrt{\frac{X}{n}}\right)\right)<\mathbb{P}\left(x \leq \sqrt{\frac{a X}{\pi n}}\right)+\mathbb{P}\left(x \geq \sqrt{\frac{b X}{\pi n}}\right)$,

where $0<a<(2 e)^{-1}$ and $b>2 e$. The $\mathbb{P}\left(x \leq \sqrt{\frac{a X}{n}}\right)$ satisfies

$$
\begin{aligned}
& \mathbb{P}\left(x \leq \sqrt{\frac{a X}{\pi n}}\right) \\
= & \sum_{k=X}^{n} \mathbb{P}\left(\text { There are } k \text { nodes within } \sqrt{\frac{a X}{\pi n}} \text { away from node } i\right) \\
= & \sum_{k=X}^{n} \frac{n !}{k !(n-k) !}\left(\frac{a X}{n}\right)^{k}\left(1-\frac{a X}{n}\right)^{n-k} .
\end{aligned}
$$

Denoting $g(k)=\frac{n !}{k !(n-k) !}\left(\frac{a X}{n}\right)^{k}\left(1-\frac{a X}{n}\right)^{n-k}$, based on Lemma 3, there must be $g(X)<c_{1}\left(a e^{1-a}\right)^{X}$. Furthermore, the ratio of $g(k)$ and $g(k+1)$ can be represented as

$$
\frac{g(k)}{g(k+1)}=\frac{\frac{n !}{k !(n-k) !}\left(\frac{a X}{n}\right)^{k}\left(1-\frac{a X}{n}\right)^{n-k}}{\frac{n !}{(k+1) !(n-k-1) !}\left(\frac{a X}{n}\right)^{k+1}\left(1-\frac{a X}{n}\right)^{n-k-1}}>\frac{k+1}{a X} .
$$

Thus, for any $k>X, g(k)$ satisfies

$$
g(k)<\frac{a X}{k} g(k-1)<\cdots<\frac{(a X)^{k-X} X !}{k !} g(X) .
$$

Therefore, $\mathbb{P}\left(x \leq \sqrt{\frac{a X}{n}}\right)$ in 19 can be upper-bounded as

$$
\begin{aligned}
\mathbb{P}\left(x \leq \sqrt{\frac{a X}{\pi n}}\right) & <\sum_{k=X}^{n} \frac{(a X)^{k-X} X !}{k !} g(X) \\
& \stackrel{(a)}{<} c_{3} \sum_{k=X}^{n} \frac{(a X)^{k-X}\left(\frac{X}{e}\right)^{X} \sqrt{X}}{\left(\frac{k}{e}\right)^{k} \sqrt{k}} g(X) \\
& =c_{3} \sum_{k=X}^{n}\left(\frac{a X e}{k}\right)^{k}\left(\frac{1}{a e}\right)^{X} \sqrt{\frac{X}{k}} g(X) \\
& <c_{3} g(X) \sum_{k=X}^{n}(a e)^{k-X}<2 c_{3} g(X),
\end{aligned}
$$

(a) holds due to 17. For $\mathbb{P}\left(x \geq \sqrt{\frac{b X}{\pi n}}\right)$, we denote $g^{*}(k)=$ $\frac{n !}{k !(n-k) !}\left(\frac{b X}{n}\right)^{k}\left(1-\frac{b X}{n}\right)^{n-k}$ and $\mathbb{P}\left(x \geq \sqrt{\frac{b X}{\pi n}}\right)<2 c_{3} g^{*}(k)$ can be proved in the similar way as above.

Hence, the probability that the distance between node $i$ and $j$ does not satisfy $x=\Theta\left(\sqrt{\frac{X}{n}}\right)$ can be represented as

$$
1-\mathbb{P}\left(x=\Theta\left(\sqrt{\frac{X}{n}}\right)\right)<2 c_{3} c_{1}\left(a e^{1-a}\right)^{X}+2 c_{3} c_{2}\left(b e^{1-b}\right)^{X} .
$$


It should be noticed that $c_{1}\left(a e^{1-a}\right)^{X}$ and $c_{2}\left(b e^{1-b}\right)^{X}$ are the monotonic functions of $a$ and $b$ with lower-bound 0 , respectively. For any given constant $c>0$, there must exist $a$ and $b$ satisfying $c_{3} c_{1}\left(a e^{1-a}\right)^{X}+c_{3} c_{2}\left(b e^{1-b}\right)^{X}<c$. Therefore, based on the definition of $o(\cdot)$, the following equation can be obtained.

$$
1-\mathbb{P}\left(x=\Theta\left(\sqrt{\frac{X}{n}}\right)\right)=o(1),
$$

which means that the $x=\Theta\left(\sqrt{\frac{X}{n}}\right)$ with probability 1 when $n$ goes to infinity.

To derive the $f_{n}(\mathbf{x})$ in Lemma 2, we first show the distribution of rank of $i$ 's friends when it has $q_{i}$ friends in the next Lemma. Afterwards, we derive the PDF in Theorem 1 based on the relation between rank and geographical position.

Lemma 5 If $\mathrm{i}$ is a source node with $q_{i}$ destinations, and $\mathrm{j}$ is one of them. The probability that $\operatorname{Rank}_{i}(j)=X$ satisfies

$$
\mathbb{P}\left\{\operatorname{Rank}_{i}(j)=X\right\}=\Theta\left(\frac{1}{q_{i}+n^{1-\alpha} X^{\alpha}}\right),
$$

when $0 \leq \alpha<1$, and

$$
\mathbb{P}\left\{\operatorname{Rank}_{i}(j)=X\right\}=\Theta\left(\frac{1}{q_{i}+X C_{1}\left(q_{i}, n\right)}\right),
$$

when $\alpha=1$ and $C_{1}\left(q_{i}, n\right)$ is given in (45), and

$$
\mathbb{P}\left\{\operatorname{Rank}_{i}(j)=X\right\}=\Theta\left(\frac{1}{q_{i}+q_{i}^{1-\alpha} X^{\alpha}}\right),
$$

when $\alpha>1$.

Proof: We sort the nodes based on the rank respects to $i$ as $v_{1}, \cdots, v_{n-1}$, where $\operatorname{Rank}_{i}\left(v_{X}\right)=X$. The set of destinations of $i$ is denoted as $Q_{i}$, where $\left|Q_{i}\right|=q_{i}$. Therefore, the probability that $\operatorname{Rank}_{i}(j)=X$ is

$$
\mathbb{P}\left\{\operatorname{Rank}_{i}(j)=X\right\}=\mathbb{P}\left\{j=v_{X} \mid v_{X} \in Q_{i}\right\} \mathbb{P}\left\{v_{X} \in Q_{i}\right\},
$$

which is shown in [9]. In particular, $\mathbb{P}\left\{j=v_{X} \mid v_{X} \in Q_{i}\right\}=\frac{1}{q_{i}}$ and $\mathbb{P}\left\{v_{X} \in Q_{i}\right\}$ can be represented as

$$
\mathbb{P}\left\{v_{X} \in Q_{i}\right\}=\frac{\frac{1}{H_{n} X^{\alpha}} \sum_{1 \leq i_{1}<\cdots<i_{q_{i}-1} \leq n, i_{h} \neq X} \prod_{h=1}^{q_{i}-1} \frac{1}{H_{n} i_{h}^{\alpha}}}{\sum_{1 \leq i_{1}<\cdots<i_{q_{i}} \leq n} \prod_{h=1}^{q_{i}} \frac{1}{H_{n} i_{h}^{\alpha}}} .
$$

We define $\sigma_{\bar{X}, q_{i}-1}=\sum_{1 \leq i_{1}<\cdots<i_{q_{i}-1} \leq n, i_{h} \neq X} \prod_{h=1}^{q_{i}-1} \frac{1}{i_{h}^{\alpha}}$ and hence

$$
\begin{aligned}
& \sum_{1 \leq i_{1}<\cdots<i_{q_{i}} \leq n} \prod_{h=1}^{q_{i}} \frac{1}{i_{h}^{\alpha}} \\
= & \sum_{1 \leq i_{1}<\cdots<i_{q_{i}} \leq n, \exists h: i_{h}=X} \prod_{h=1}^{q_{i}} \frac{1}{i_{h}^{\alpha}}+\sum_{1 \leq i_{1}<\cdots<i_{q_{i}} \leq n, i_{h} \neq X} \prod_{h=1}^{q_{i}} \frac{1}{i_{h}^{\alpha}} \\
= & \frac{1}{X^{\alpha}} \sigma_{\bar{X}, q_{i}-1}+\sigma_{\bar{X}, q_{i}} .
\end{aligned}
$$

Denoting $C_{\sigma}\left(X, q_{i}, n\right)$ as

$$
C_{\sigma}\left(X, q_{i}, n\right)=q_{i} \frac{\sigma_{\bar{X}, q_{i}}}{\sigma_{\bar{X}, q_{i}-1}},
$$

and thus $\mathbb{P}\left\{\operatorname{Rank}_{i}(j)=X\right\}$ can be transformed as

$$
\mathbb{P}\left\{\operatorname{Rank}_{i}(j)=X\right\}=\frac{1}{q_{i}+C_{\sigma}\left(X, q_{i}, n\right) X^{\alpha}} .
$$

Then, we prove that the order of $C\left(X, q_{i}, n\right)$ is independent from $X$ when $C\left(X, q_{i}, n\right) X^{\alpha}=\omega\left(q_{i}\right)$ (i.e., for any constant $c_{4}$ there must be $\left.C\left(X, q_{i}, n\right) X^{\alpha}>c_{4} q_{i}\right)$. We arbitrarily select two integers $X_{a}$ and $X_{b}$. Based on (32) and $C\left(X, q_{i}, n\right) X^{\alpha}>$ $c_{4} q_{i}$ we can obtain

$$
\begin{gathered}
\sigma_{\overline{X_{1} X_{2}}, q_{i}}+\frac{\sigma_{\overline{X_{1} X_{2}}, q_{i}-1}}{X_{2}^{\alpha}}>c_{4}\left(\frac{\sigma_{\overline{X_{1} X_{2}}, q_{i}-1}}{X_{1}^{\alpha}}+\frac{\sigma_{\overline{X_{1} X_{2}}, q_{i}-2}}{X_{2}^{\alpha} X_{1}^{\alpha}}\right), \\
\sigma_{\overline{X_{1} X_{2}}, q_{i}}+\frac{\sigma_{\overline{X_{1} X_{2}}, q_{i}-1}}{X_{1}^{\alpha}}>c_{4}\left(\frac{\sigma_{\overline{X_{1} X_{2}}, q_{i}-1}}{X_{2}^{\alpha}}+\frac{\sigma_{\overline{X_{1} X_{2}}, q_{i}-2}}{X_{2}^{\alpha} X_{1}^{\alpha}}\right) .
\end{gathered}
$$

If $c_{4}>1, \sigma_{\overline{X_{1} X_{2}}, q_{i}}$ can be lower-bounded as

$$
\sigma_{\overline{X_{1} X_{2}}, q_{i}}>\frac{\left(c_{4}-1\right) \sigma_{\overline{X_{1} X_{2}}, q_{i}-1}}{2 X_{2}^{\alpha}}+\frac{\left(c_{4}-1\right) \sigma_{\overline{X_{1} X_{2}}, q_{i}-1}}{2 X_{1}^{\alpha}} .
$$

According to the Newton's inequality in [22] that for any constant $c_{5}>0, \sigma_{\overline{X_{1} X_{2}}, q_{i}-1}$ can be lower-bounded as

$$
\sigma_{\overline{X_{1} X_{2}}, q_{i}-1}>\frac{c_{5} \sigma_{\overline{X_{1} X_{2}}, q_{i}} \sigma_{\overline{X_{1} X_{2}}, q_{i}-2} .}{\sigma_{\overline{X_{1} X_{2}}, q_{i}-1}} .
$$

Therefore, the relation between $\sigma_{\overline{X_{1} X_{2}}, q_{i}-1}$ and $\frac{\sigma_{\overline{X_{1} X_{2}, q_{i}-2}}}{X_{2}^{\alpha}}$ can be derived as

$$
\begin{aligned}
& \sigma_{\overline{X_{1} X_{2}}, q_{i}-1}>\frac{c_{5} \sigma_{\overline{X_{1} X_{2}}, q_{i}} \sigma_{\overline{X_{1} X_{2}}, q_{i}-2}}{\sigma_{\overline{X_{1} X_{2}}, q_{i}-1}} \\
& =\frac{c_{5}}{X_{2}^{\alpha}} \frac{\sigma_{\overline{X_{1} X_{2}}, q_{i}} \sigma_{\overline{X_{1} X_{2}}, q_{i}-2} \frac{\sigma_{\overline{X_{1} X_{2}}, q_{i}-1}^{X_{2}^{\alpha}}}{X_{2}^{\alpha}}}{c_{5}\left(c_{1}-1\right) \sigma_{X_{1} X_{2}}} \\
& >\frac{c_{5}\left(c_{4}-1\right) \sigma_{\overline{X_{1} X_{2}}, q_{i}-2}}{X_{2}^{\alpha}} .
\end{aligned}
$$

Thus, we calculate the bound of $C\left(X_{1}, q_{i}, n\right)$ as follows

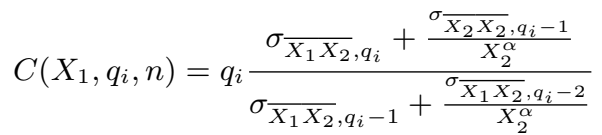

$$
\begin{aligned}
& >\frac{q_{i} \sigma_{\overline{X_{1} X_{2}}, q_{i}}}{\left(\frac{1}{c_{5}\left(c_{4}-1\right)}+1\right) \sigma_{\overline{X_{1} X_{2}}, q_{i}-1}}, \\
& C\left(X_{1}, q_{i}, n\right)<\frac{\left(\frac{1}{c_{5}\left(c_{4}-1\right)}+1\right) q_{i} \sigma_{\overline{X_{1} X_{2}}, q_{i}} .}{\sigma_{\overline{X_{1} X_{2}}, q_{i}-1}} .
\end{aligned}
$$

Therefore, it is obvious that

$$
C\left(X_{1}, q_{i}, n\right)=\Theta\left(\frac{q_{i} \sigma_{\overline{X_{1} X_{2}}, q_{i}}}{\sigma_{\overline{X_{1} X_{2}}, q_{i}-1}}\right) .
$$

Similarly,

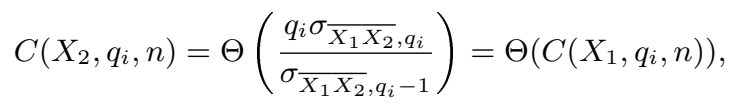

which means that the order of $C\left(X, q_{i}, n\right)$ is independent from $X$ when $C\left(X, q_{i}, n\right) X^{\alpha}=\omega\left(q_{i}\right)$. Hence, we denote $C\left(X, q_{i}, n\right)$ as $c_{X} C\left(q_{i}, n\right)$ where $c_{X}$ may be related to $X, q_{i}$. 
According to equation (38), the upper-bound and lower-bound of $c_{X}$ are $\left(\frac{1}{c_{5}\left(c_{4}-1\right)}+1\right)^{2}$ and $\left(\frac{1}{c_{5}\left(c_{4}-1\right)}+1\right)^{-2}$, respectively. Since $c_{4}>1$ and $c_{5}>0$ are arbitrary constants, we obtain that $c_{X}=1$. Thus, (33) can be rewritten as

$$
\mathbb{P}\left\{\operatorname{Rank}_{i}(j)=X\right\}=\frac{1}{q_{i}+C\left(q_{i}, n\right) X^{\alpha}},
$$

Now we analyze $\mathbb{P}\left\{\operatorname{Rank}_{i}(j)=X\right\}$ for three cases.

Case 1: $0 \leq \alpha<1$. In this case, because of 29) and 30, the sum of (41) is 1 , which means that

$$
\sum_{X=1}^{n} \frac{1}{q_{i}+C\left(q_{i}, n\right) X^{\alpha}}=\frac{c_{6} q_{i}^{\frac{1-\alpha}{\alpha}}}{C^{\frac{1}{\alpha}}\left(q_{i}, n\right)}+\frac{c_{6} n^{1-\alpha}}{C\left(q_{i}, n\right)}=1,
$$

where $1 / 2<c_{6}<1$ is constant. Therefore,

$$
c_{6} n^{1-\alpha}<C\left(q_{i}, n\right)<2 c_{6} n^{1-\alpha}
$$

which shows that $C\left(q_{i}, n\right)=c_{X}^{\prime} n^{1-\alpha}$, where $c_{X}^{\prime}$ is related to $c_{X}$ and $1 / 2<c_{X}^{\prime}<2$. Hence, 33 can be rewritten as

$$
\mathbb{P}\left\{\operatorname{Rank}_{i}(j)=X\right\}=\frac{1}{q_{i}+c_{X}^{\prime} n^{1-\alpha} X^{\alpha}}=\Theta\left(\frac{1}{q_{i}+n^{1-\alpha} X^{\alpha}}\right) .
$$

Case 2: $\alpha=1$. We derive $C\left(q_{i}, n\right)$ and $\mathbb{P}\left\{\operatorname{Rank}_{i}(j)=X\right\}$ in the same way as in case 1 . It can be obtained that

$$
C\left(q_{i}, n\right)=C_{1}\left(q_{i}, n\right)= \begin{cases}\Theta\left(\log \frac{n}{q_{i}}\right) & \lim _{n \rightarrow \infty} \frac{n}{q_{i}}>1, \\ \Theta(1) & \lim _{n \rightarrow \infty} \frac{n}{q_{i}}=1,\end{cases}
$$

and

$$
\mathbb{P}\left\{\operatorname{Rank}_{i}(j)=X\right\}=\frac{1}{q_{i}+c_{X}^{\prime \prime} X C_{1}\left(q_{i}, n\right)}=\Theta\left(\frac{1}{q_{i}+X C_{1}\left(q_{i}, n\right)}\right),
$$

where $1 / 2<c_{X}^{\prime \prime}<2$.

Case 3: $\alpha>$ 1. $C\left(q_{i}, n\right)$ and $\mathbb{P}\left\{\operatorname{Rank}_{i}(j)=X\right\}$ can also be obtained in the similar way, and the results are $C\left(q_{i}, n\right)=$ $\Theta\left(q_{i}^{1-\alpha}\right)$ and

$$
\mathbb{P}\left\{\operatorname{Rank}_{i}(j)=X\right\}=\frac{1}{q_{i}+c_{X}^{\prime \prime \prime} q_{i}^{1-\alpha} X^{\alpha}}=\Theta\left(\frac{1}{q_{i}+q_{i}^{1-\alpha} X^{\alpha}}\right),
$$

where $1 / 2<c_{X}^{\prime \prime \prime}<2$.

Based on Lemma 4 and 5, the PDF of destinations is obtained in the following theorem in polar coordinates.

Theorem 1 If node $\mathrm{i}$ is a source, and it selects $q_{i}$ destinations based on (6), the location of each destination follows the distribution

$$
f_{n}(x, \theta)=\Theta\left(\frac{n}{q_{i}+n x^{2 \alpha}}\right),
$$

when $0 \leq \alpha<1$, and

$$
f_{n}(x, \theta)=\Theta\left(\frac{n}{q_{i}+n x^{2} C_{1}\left(q_{i}, n\right)}\right),
$$

when $\alpha=1$, and

$$
f_{n}(x, \theta)=\Theta\left(\frac{n}{q_{i}+q_{i}^{1-\alpha} n^{\alpha} x^{2 \alpha}}\right),
$$

when $\alpha>1$.

Proof: Firstly, we analyze the PDF for the case $0 \leq \alpha<$ 1. For node $i$ and its destination $j$, denoting the Cumulative Distribution Functions (CDF) of $d(i, j)$ as $F_{i}(x)$, it can be represented as

$$
\begin{aligned}
F_{i}(x) & =\mathbb{P}\{d(i, j)<x\} \\
& =\sum_{X=1}^{n} \mathbb{P}\left\{d(i, j)<x \mid \operatorname{Rank}_{i}(j)=X\right\} \mathbb{P}\left\{\operatorname{Rank}_{i}(j)=X\right\} .
\end{aligned}
$$

Hence, the PDF can be expressed as

$$
\begin{aligned}
f_{n}(x, \theta) & =\frac{d F_{i}(x)}{2 \pi x d x} \\
& =\sum_{R=1}^{n} \frac{d \mathbb{P}\left\{d(i, j)<x \mid \operatorname{Rank}_{i}(j)=k\right\}}{2 \pi x d x} \cdot \mathbb{P}\left\{\operatorname{Rank}_{i}(j)=R\right\} \\
& =\sum_{X=1}^{n} \frac{d \sum_{k=X}^{n} \frac{n !}{k !(n-k) !}\left(\pi x^{2}\right)^{k}\left(1-\pi x^{2}\right)^{n-k}}{2 \pi\left(q_{i}+c_{X}^{\prime} n^{1-\alpha} X^{\alpha}\right) x d x} \\
& \stackrel{(a)}{=} \sum_{X=1}^{n} \frac{d I_{\pi x^{2}(X, n-X+1)}}{2 \pi\left(q_{i}+c_{X}^{\prime} n^{1-\alpha} X^{\alpha}\right) x d x},
\end{aligned}
$$

where $I_{x}(a, b)$ is the regularized incomplete beta function and $(a)$ holds due to equation (8.17.5) in [23]. If $q_{i}>$ $(2 e \pi)^{\alpha} n x^{2 \alpha}$, the PDF is as follow

$$
\begin{aligned}
& f_{n}(x, \theta) \\
= & \sum_{X=1}^{n} \frac{n !}{\frac{(X-1) !(n-X) !}{\left.2 \pi x^{2}\right)^{X-1}\left(1-\pi x^{2}\right)^{n-X} n^{1-\alpha} X^{\alpha}}} \\
< & \frac{n}{2 \pi q_{i}} \sum_{X=1}^{X_{0}} \frac{(n-1) !}{(X-1) !(n-X) !}\left(\pi x^{2}\right)^{X-1}\left(1-\pi x^{2}\right)^{n-X}+ \\
& n^{\alpha} \sum_{X=X_{0}+1}^{n} \frac{(n-1) !}{2 \pi c_{X}^{\prime}(X-1) !(n-X) ! X^{\alpha}}\left(\pi x^{2}\right)^{X-1}\left(1-\pi x^{2}\right)^{n-X} \\
= & \frac{n}{2 \pi q_{i}} \sum_{X=1}^{X_{0}} \frac{(n-1) !}{(X-1) !(n-X) !}\left(\frac{a X_{0}}{n}\right)^{X-1}\left(1-\frac{a X_{0}}{n}\right)^{n-X}+ \\
& n^{\alpha} \sum_{X=X_{0}+1}^{n} \frac{(n-1) !}{2 \pi c_{X}^{\prime}(X-1) !(n-X) ! X^{\alpha}}\left(\frac{a X_{0}}{n}\right)^{X-1}\left(1-\frac{a X_{0}}{n}\right)^{n-X} \\
& (a) \\
< & \frac{n}{2 \pi q_{i}}+\frac{c_{1}\left(a e^{1-a}\right)^{X_{0}+1} n^{\alpha}}{2 \pi c_{X}^{\prime} X_{0}^{\alpha}} \\
< & \frac{n}{\pi q_{i}},
\end{aligned}
$$

where $X_{0}=\left|\frac{q_{i}^{\frac{1}{\alpha}}}{\pi n^{\frac{1-\alpha}{\alpha}}}\right|, a=\frac{n x^{2}}{X_{0}}<(2 e)^{-1}$ and $\underline{c_{X}^{\prime}}$ is the lower-bound of $c_{X}^{\prime}$, which equals to $1 / 2$. It should be noticed that the $f_{n}(x, \theta)$ is uniform for different directions, and thus there is no $\theta$ in the expression of $f_{n}(x, \theta)$. (a) in 53) holds due to the proof of Lemma 4 which shows that

$$
\sum_{X=X_{0}+1}^{n} \frac{(n-1) !\left(\frac{a X_{0}}{n}\right)^{X-1}\left(1-\frac{a X_{0}}{n}\right)^{n-X}}{(X-1) !(n-X) !}<c_{1}\left(a e^{1-a}\right)^{X_{0}+1}
$$

when $a<(2 e)^{-1}$. In the same way, it can also be proved that

$$
f_{n}(x, \theta)>\frac{n}{2 \pi q_{i}}-\frac{c_{1}\left(a e^{1-a}\right)^{X_{0}+1} n^{\alpha}}{\pi \overline{c_{X}^{\prime}} X_{0}^{\alpha}}>\frac{n}{8 \pi q_{i}},
$$


where $\overline{c_{X}^{\prime}}$ is the upper-bound of $c_{X}^{\prime}$, which equals to 2 . Hence, $f_{n}(x, \theta)=\Theta\left(\frac{n}{q_{i}}\right)$ is proved when $q_{i}=\omega\left(n x^{2 \alpha}\right)$. Moreover, if $q_{i}=O\left(n x^{2 \alpha}\right)$, after some similar mathematical manipulations, the $f_{n}(x, \theta)$ can be represented as

$$
f_{n}(x, \theta)=\Theta\left(x^{-2 \alpha}\right) .
$$

Consequently, the PDF for $0 \leq \alpha<1$ is

$$
f_{n}(x, \theta)=\Theta\left(\max \left\{x^{-2 \alpha}, \frac{n}{q_{i}}\right\}\right)=\Theta\left(\frac{n}{q_{i}+n x^{2 \alpha}}\right),
$$

Moreover, the $f_{n}(x, \theta)$ for $\alpha \geq 1$ can be obtained in the same way, and the results are shown in 49 and 50 .

According to Lemma 2 and Theorem 1 1 , we analyze the expression of the $\overline{\|E M S T\|}$ in the following theorem.

Theorem 2 When $\alpha=1$, the expectation of the bound $\|E M S T\|$ in 11) satisfies

$$
\overline{\|E M S T\|}= \begin{cases}\Theta\left(\sqrt{\frac{q_{i}}{C_{1}\left(q_{i}, n\right)}}\right) & q_{i}=\omega(\log n), \\ O\left(\frac{q_{i}}{\log n}\right) & q_{i}=O(\log n) .\end{cases}
$$

Furthermore, when $0 \leq \alpha<1$, the expectation of the bound $\overline{\|E M S T\|}$ in (11) satisfies

$$
\overline{\|E M S T\|}=\Theta\left(\sqrt{q_{i}}\right) .
$$

Finally, when $\alpha>1$, the expectation of the bound $\overline{\|E M S T\|}$ in (11) satisfies

$$
\overline{\|E M S T\|}=\left\{\begin{array}{lc}
O\left(q_{i}^{\alpha} n^{1-\alpha}\right) & q_{i}=O\left(n^{\frac{\alpha-1}{\alpha}}\right), 1<\alpha \leq \frac{3}{2}, \\
\tilde{\Theta}\left(q_{i}^{\frac{\alpha}{2}} n^{\frac{1-\alpha}{2}}\right) & q_{i}=\omega\left(n^{\frac{\alpha-1}{\alpha}}\right), 1<\alpha \leq \frac{3}{2}, \\
O\left(q_{i}^{\frac{\alpha}{2 \alpha-2}} n^{-\frac{1}{2}}\right) & q_{i}=O\left(n^{\frac{\alpha-1}{\alpha}}\right), \frac{3}{2}<\alpha<2, \\
\tilde{\Theta}\left(q_{i}^{\frac{\alpha}{2}} n^{\frac{1-\alpha}{2}}\right) & q_{i}=\omega\left(n^{\frac{\alpha-1}{\alpha}}\right), \frac{3}{2}<\alpha<2, \\
\tilde{\Theta}\left(\frac{q_{i}}{\sqrt{n}}\right) & \alpha \geq 2 .
\end{array}\right.
$$

Proof: Case 1: $\alpha=1$. Based on Lemma 2 and Theorem 1. the $\overline{\|E M S T\|}$ can be derived for different value of $q_{i}$. If $q_{i}=\omega(\log n)$, we prove that the $\overline{\|E M S T\|}$ can be calculated by (11) first. The network can be separated into some small square regions as in Lemma 2, and we assume that the side length of the region $\xi$, which is $d$ away from the source, is $r$, where $d>r$. The number of destinations in this region is lower-bounded as

$$
\int_{\xi} f_{n}(x, \theta) d \xi>\frac{q_{i}}{2} \int_{0}^{\frac{r}{d}} \int_{d-\frac{r}{2}}^{d+\frac{r}{2}} x f_{n}(x, \theta) d x d \theta=\frac{c_{8} q_{i} r^{2}}{d^{2} C_{1}\left(q_{i}, n\right)},
$$

where $d \geq \sqrt{\frac{q_{i}}{n C_{1}\left(q_{i}, n\right)}}$, and $\frac{1}{8 \pi}<c_{8}<\frac{1}{2 \pi}$ is constant. It is necessary to guarantee that (61) is no less than 1 , and therefore, $r \geq d \sqrt{\frac{C_{1}\left(q_{i}, n\right)}{c_{8} q_{i}}}$. Since a small $r$ is needed for $g_{n}(x, \theta)$ which satisfies Condition 1 in Lemma 2, $r$ can be represented as

$$
r=d \sqrt{\frac{C_{1}\left(q_{i}, n\right)}{c_{8} q_{i}}} .
$$

Moreover, $d>r$ because $q_{i}=\omega(\log n)=\omega\left(C_{1}\left(q_{i}, n\right)\right)$. Since $d>r$ and $r \propto d^{\alpha}$, there are at most $2^{\alpha}+1$ adjacent parts at each side of $\xi_{i}$. Considering the fact that $\xi_{i}$ has only 4 sides, the $\zeta$ in the Theorem 2 is no greater than $2^{\alpha+2}+4$, which is a constant. Thus the Conditions in Lemma 2 are satisfied. Similarly, the Conditions in Lemma 2 also hold in the case $0 \leq \alpha<1$ and $\alpha>1$, and we do not show it in the following proof for brevity.

We define $g_{n}(x, \theta)=\frac{1}{r^{2}} \int_{\xi} x f_{n}(x, \theta) d x d \theta$ when $(x, \theta)$ is in $\xi$, where $\xi$ is a square region with side length $r$. If $d \geq$ $\sqrt{\frac{q_{i}}{n C_{1}\left(q_{i}, n\right)}}$, the difference between $g_{n}(x, \theta)$ and $f_{n}(x, \theta)$ in $\xi$ can be derived as

$$
\begin{aligned}
\int_{\xi}\left|f_{n}(x, \theta)-g_{n}(x, \theta)\right| d \xi & <\int_{0}^{\frac{r}{d}} \int_{d-\frac{r}{2}}^{d+\frac{r}{2}}\left|f_{n}(x, \theta)-g_{n}(x, \theta)\right| x d x d \theta \\
& =\frac{c_{9} r^{3}}{d^{3} C_{1}\left(q_{i}, n\right)}
\end{aligned}
$$

where $\frac{1}{8 \pi}<c_{9}<\frac{1}{\pi}$ is constant. Thus, according to 53 and (55), the condition 1 of Lemma 2 can be proved as follow

$$
\begin{aligned}
& \int_{\Psi}\left|f_{n}(x, \theta)-g_{n}(x, \theta)\right| d \xi \\
= & \int_{0}^{2 \pi} \int_{\sqrt{\frac{q_{i}}{n C_{1}\left(q_{i}, n\right)}}\left|f_{n}(x, \theta)-g_{n}(x, \theta)\right| x d x d \theta}^{1} \\
& +\int_{0}^{2 \pi} \int_{0}^{\sqrt{\frac{q_{i}}{n C_{1}\left(q_{i}, n\right)}}\left|f_{n}(x, \theta)-g_{n}(x, \theta)\right| x d x d \theta} \\
\stackrel{(a)}{<} & \sum_{d} \frac{2 \pi d}{r} \int_{0}^{\frac{r}{d}} \int_{d-\frac{r}{2}}^{d+\frac{r}{2}}\left|f_{n}(x, \theta)-g_{n}(x, \theta)\right| x d x d \theta \\
& +\int_{0}^{2 \pi} \int_{0}^{\sqrt{\frac{q_{i}}{n C_{1}\left(q_{i}, n\right)}}} \frac{c_{1}\left(a e^{1-a}\right)^{X_{0}+1}\left(\overline{c_{X}^{\prime}}+\underline{c_{X}^{\prime}}\right) n}{2 \pi \overline{c_{X}^{\prime}} c_{X}^{\prime} q_{i}} x d x d \theta \\
< & \sum_{d} \frac{2 \pi c_{9} r^{2}}{d^{2} C_{1}\left(q_{i}, n\right)}+\frac{c_{1}\left(a e^{1-a}\right)^{X_{0}+1}\left(\overline{c_{X}^{\prime}}+\underline{c_{X}^{\prime}}\right)}{\overline{c_{X}^{\prime}}}, \underline{c_{X}^{\prime}}
\end{aligned}
$$

(a) holds because there are less than $\frac{2 \pi d}{r}$ regions with side length $r$ and $d$ from the source. Moreover, considering the relation between $d$ and $r$ in 62 and the maximum value of $d$ is $\frac{1}{2}$, 64, can be bounded by

$$
\begin{aligned}
& \sum_{d} \frac{2 \pi c_{9} r^{2}}{d^{2} C_{1}\left(q_{i}, n\right)}+\frac{c_{1}\left(a e^{1-a}\right)^{X_{0}+1}\left(\overline{c_{X}^{\prime}}+\underline{c_{X}^{\prime}}\right)}{\overline{c_{X}^{\prime}} c_{X}^{\prime}} \\
< & \frac{4 \pi c_{9} \log n}{\sqrt{c_{8} q_{i} C_{1}\left(q_{i}, n\right)}}+\frac{c_{1}\left(a e^{1-a}\right)^{X_{0}+1}\left(\overline{c_{X}^{\prime}}+\underline{c_{X}^{\prime}}\right)}{\overline{c_{X}^{\prime}} c_{X}^{\prime}} \\
= & o(1) .
\end{aligned}
$$

Therefore, the two conditions in Lemma 2 are satisfied. We denote that $x^{\dagger}=\sqrt{\frac{q_{i}}{n C_{1}\left(q_{i}, n\right)}}$ and the $\overline{\|E M S T\|}$ is as

$$
\begin{aligned}
\overline{\|E M S T\|} & <\overline{c_{10}} c(d) \sqrt{q_{i}} \int_{0}^{2 \pi} \int_{0}^{1} x \sqrt{\frac{n}{q_{i}+n C_{1}\left(q_{i}, n\right) x^{2}}} d x d \theta \\
& <2 \overline{c_{10}} c(d) \pi \sqrt{q_{i}}\left[\int_{0}^{x^{\dagger}} \frac{x \sqrt{n}}{\sqrt{q_{i}}} d x+\int_{x^{\dagger}}^{1} \sqrt{\frac{1}{C_{1}\left(q_{i}, n\right)}} d x\right] \\
& <2 \overline{c_{10}} c(d) \pi \sqrt{\frac{q_{i}}{C_{1}\left(q_{i}, n\right)}},
\end{aligned}
$$


where $\overline{c_{10}}$ is constant according to the proof of Theorem 1 Similarly, 67 can also be proved.

$$
\overline{\|E M S T\|}>\frac{c_{10} c(d) \pi}{2} \sqrt{\frac{q_{i}}{C_{1}\left(q_{i}, n\right)}},
$$

where $\underline{c_{10}}$ is constant according to Theorem 1. Thus, $\overline{\|E M S T\|}=\Theta\left(\sqrt{\frac{q_{i}}{C_{1}\left(q_{i}, n\right)}}\right)$.

If $q_{i}=\Theta(1)$, (11) cannot be applied. However, the $\overline{\|E M S T\|}$ is upper-bounded by the sum distance between $i$ and its $q_{i}$ friends and lower-bounded by the distance between $i$ and one of its friends. Therefore, the order of $\overline{\|E M S T\|}$ can be expressed as

$$
\overline{\|E M S T\|}=\Theta\left(\frac{1}{\log n}\right) .
$$

If $q_{i}=\omega(1)$ and $q_{i}=O(\log n)$, it is obvious that the two conditions in Lemma 2 cannot be satisfied simultaneously based on 61 to 65. Therefore, 11 cannot be adopted in this case, and we give the upper-bound of $\overline{\|E M S T\|}$ as follow

$$
\overline{\|E M S T\|}=O\left(\frac{q_{i}}{\log n}\right)
$$

which is the sum length of $q_{i}$ direct links. Consequently, equation 58, is proved.

Case 2: $\mathbf{0} \leq \alpha<\mathbf{1}$. We also derive the $\overline{\|E M S T\|}$ based on $q_{i}$. The network is separated into some small square regions as in Lemma 2. We assume that the side length of the region $\xi$, which is $d$ away from the source, is $r$, and $d>r$. Similar to the proof of case 1, the two conditions in Lemma 2 are satisfied for the region $x<\left(\frac{q_{i}}{n}\right)^{\frac{1}{2 \alpha}}$. Therefore, we only focus on the case $x>\left(\frac{q_{i}}{n}\right)^{\frac{1}{2 \alpha}}$. The number of destinations in the region with side length $r$ and $d>\left(\frac{q_{i}}{n}\right)^{\frac{1}{2 \alpha}}$ is lower-bounded by

$$
\frac{q_{i}}{2} \int_{0}^{\frac{r}{d}} \int_{d-\frac{r}{2}}^{d+\frac{r}{2}} x f_{n}(x, \theta) d x d \theta=\frac{c_{11} q_{i} r^{2}}{d^{2 \alpha}}
$$

where $\frac{1}{8 \pi}<c_{11}<\frac{1}{2 \pi}$. Since it is necessary that $\frac{c_{11} q_{i} r^{2}}{d^{2} \alpha} \geq 1$, the $r$ can be bounded as $r \geq \frac{d^{\alpha}}{\sqrt{c_{11} q_{i}}}$. Thus, $r$ is selected as

$$
r=\frac{d^{\alpha}}{\sqrt{c_{11} q_{i}}}
$$

Moreover, $d>r$, i.e., $d>\left(c_{11} q_{i}\right)^{\frac{1}{2 \alpha-2}}$. We define $g_{n}(x, \theta)=$ $\frac{1}{r^{2}} \int_{\xi} x f_{n}(x, \theta) d x d \theta$, where $\xi$ is a square region with side length $r$. Thus, if $\left(c_{11} q_{i}\right)^{\frac{1}{2 \alpha-2}}<\left(\frac{q_{i}}{n}\right)^{\frac{1}{2 \alpha}}, \int_{\Psi} \mid f_{n}(x, \theta)-$ $g_{n}(x, \theta) \mid d \xi=o(1)$ can be proved in the similar way as in 63. to 65 , and therefore the $f_{n}(x, \theta)$ satisfies two conditions in Lemma 2 .

If $\left(c_{11} q_{i}\right)^{\frac{1}{2 \alpha-2}}>\left(\frac{q_{i}}{n}\right)^{\frac{1}{2 \alpha}}$, we will propose another PDF $f_{n}^{\prime}(x, \theta)$, which satisfies the two conditions and its corresponding $\overline{\|E M S T\|}$ is the same as $f_{n}(x, \theta)$ in order sense. The number of destinations within the ring $\left(\frac{q_{i}}{n}\right)^{\frac{1}{2 \alpha}}<d<\left(c_{11} q_{i}\right)^{\frac{1}{2 \alpha-2}}$ is upper-bounded as

$$
q_{i} \int_{0}^{2 \pi} \int_{\left(\frac{q_{i}}{n}\right)^{\frac{1}{2 \alpha}}}^{\left(c_{11} \sqrt{q_{i}}\right)^{\frac{1}{\alpha-1}}} x f_{n}(x, \theta) d x d \theta<\frac{\pi}{c_{11}(1-\alpha)} .
$$

Hence, the total length of links in this region is smaller than $\frac{\pi\left(c_{11}\right)^{\frac{2-\alpha}{\alpha-1}} q_{i}^{\frac{1}{2 \alpha-2}}}{1-\alpha}$. Thus, we define the new PDF as

$$
f_{n}^{\prime}(x, \theta)
$$

$$
= \begin{cases}f_{n}(x, \theta) & x \leq\left(\frac{q_{i}}{n}\right)^{\frac{1}{2 \alpha}}, \\ \frac{\int_{0}^{2 \pi} \int_{\left(\frac{q_{i}}{n}\right)^{\frac{1}{2 \alpha}}\left(c_{11} q_{i} \frac{1}{2 \alpha-2}\right.}^{\frac{1}{2 \alpha}} x f_{n}(x, \theta) d x d \theta}{\pi\left(c_{11} q_{i}\right)^{\frac{1}{\alpha-1}}-\pi\left(\frac{q_{i}}{n}\right)^{\frac{1}{\alpha}}} & \left(\frac{q_{i}}{n}\right)^{\frac{1}{2 \alpha}}<x<\left(c_{11} q_{i}\right)^{\frac{1}{2 \alpha-2}}, \\ f_{n}(x, \theta) & x \geq\left(c_{11} q_{i}\right)^{\frac{1}{2 \alpha-2}} .\end{cases}
$$

The difference of $\overline{\|E M S T\|}$ between $f_{n}(x, \theta)$ and $f_{n}^{\prime}(x, \theta)$ is smaller than $\frac{\pi\left(c_{11}\right)^{\frac{2-\alpha}{\alpha-1}} q_{i}^{\frac{1}{2 \alpha-2}}}{1-\alpha}$. Moreover, similar to the proof from 63 to 65 , there exists a $g_{n}(x, \theta)$ satisfying the two conditions in Lemma 2 with respect to $f_{n}^{\prime}(x, \theta)$. Hence, the $\overline{\|E M S T\|}$ of $f_{n}^{\prime}(x, \theta)$ can be derived by Lemma 2 Furthermore, since $\overline{\|E M S T\|}>1>\frac{2 \pi\left(c_{11}\right)^{\frac{2-\alpha}{\alpha-1}} q_{i}^{\frac{1}{2 \alpha-2}}}{1-\alpha}$, the order of $\overline{\|E M S T\|}$ of $f_{n}(x, \theta)$ and $f_{n}^{\prime}(x, \theta)$ are the same. Consequently, $\overline{\|E M S T\|}$ for $f_{n}(x, \theta)$ is the same as $f_{n}^{\prime}(x, \theta)$ in order sense for the case $0<\alpha<1$. Similar to 66 and 67, the result of $\overline{\|E M S T\|}$ can be represented as in (59).

Case 3: $\alpha>1$. We try to find when the two conditions in Lemma 2 are satisfied first. The network is also separated into some small square regions as above. Therefore, the number of destinations in one region $\xi$ and $d>\sqrt{\frac{q_{i}}{n}}$ is lower-bounded as

$$
q_{i} \int_{0}^{\frac{r}{d}} \int_{d-\frac{r}{2}}^{d+\frac{r}{2}} x f_{n}(x, \theta) d x d \theta>\frac{c_{12} q_{i}^{\alpha} r^{2}}{d^{2 \alpha} n^{\alpha-1}}
$$

where $\frac{1}{8 \pi}<c_{12}<\frac{1}{2 \pi}$. We select $r=\frac{n^{\frac{\alpha-1}{2}} d^{\alpha}}{\sqrt{c_{12}} q_{i}^{\frac{\alpha}{2}}}$ to ensure that (74) equals to 1 . Moreover, $d>r$ which indicates that $d<$ $\frac{\left(c_{12}\right)^{\frac{1}{2 \alpha-2}} q_{i}^{\frac{\alpha}{2 \alpha-2}}}{\sqrt{n}}$. We define $g_{n}(x, \theta)=\frac{1}{r^{2}} \int_{\xi} x f_{n}(x, \theta) d x d \theta$ when $(x, \theta)$ is in $\xi$. Thus, if $q_{i}>n^{\frac{\alpha-1}{\alpha}}$, it can be proved in the similar way as in (63) to 65 that

$$
\int_{\Psi}\left|f_{n}(x, \theta)-g_{n}(x, \theta)\right| d \xi=o(1) .
$$

Hence, when $q_{i}>n^{\frac{\alpha-1}{\alpha}}$, the two conditions in Lemma 2 are satisfied. and the $\overline{\|E M S T\|}$ can be calculated by $(11)$.

However, if $q_{i}<n^{\frac{\alpha-1}{\alpha}}$, the two conditions in Lemma 2 are no longer satisfied. In order to analyze the bound of $\|E M S T\|$, we divide the network into two groups. In the group 1, the nodes in the circle centered at the source with radius $\frac{q_{i}^{\frac{\alpha}{2 \alpha-2}}}{\sqrt{n}}$ are considered, and the nodes out of it are not considered. In the group 2, other nodes are considered. The PDFs of them are denoted as $f_{n}^{(1)}(x, \theta)$ and $f_{n}^{(2)}(x, \theta)$, respectively. The $f_{n}^{(1)}(x, \theta)$ can be derived as

$$
f_{n}^{(1)}(x, \theta)=\frac{f_{n}(x, \theta)}{\int_{0}^{2 \pi} \int_{0}^{\frac{q_{i}^{2 \alpha-2}}{\sqrt{n}}} x f_{n}(x, \theta) d x d \theta}=c_{13} f_{n}(x, \theta),
$$

for $x<\frac{q_{i}^{\frac{\alpha}{2 \alpha-2}}}{\sqrt{n}}$, and $f_{1}(x, \theta)=0$ for $x>\frac{q_{i}^{\frac{\alpha}{2 \alpha-2}}}{\sqrt{n}}$, where $c_{13} \rightarrow 1$ when $n \rightarrow \infty$. According to 63 to 65$]$, it can be 
found that the nodes in group 1 satisfy condition 1 and 2 in Lemma 2. Thus, the $\overline{\|E M S T\|}$ for group 1 can be calculated based on Lemma 2. Then we derive $f_{n}^{(2)}(x, \theta)$ as

$$
f_{n}^{(2)}(x, \theta)=\frac{f_{n}(x, \theta)}{\int_{0}^{2 \pi} \int_{\frac{q_{i} \frac{\alpha}{\sqrt{n}}}{2 \alpha-2}}^{1} x f_{n}(x, \theta) d x d \theta}>\frac{q_{i} f_{n}(x, \theta)}{\underline{c_{14}}},
$$

for $x>\frac{q_{i}^{\frac{\alpha}{2 \alpha-2}}}{\sqrt{n}}$, and $f_{2}(x, \theta)=0$ for $x<\frac{q_{i}^{\frac{\alpha}{2 \alpha-2}}}{\sqrt{n}}$, where $\underline{c_{14}}>0$ is constant according to Theorem 1 . The number of destinations in group 2 is

$$
q_{i} \int_{0}^{2 \pi} \int_{\frac{q_{i}^{2 \alpha-2}}{\sqrt{n}}}^{1} x f_{n}(x, \theta) d x d \theta<\underline{c_{14}} .
$$

And the average distance from these destinations to the source is

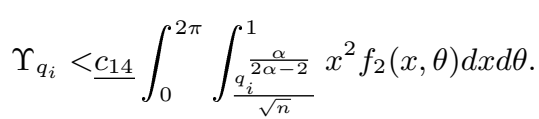

Since the total $\overline{\|E M S T\|}$ for this case is upper-bounded by the sum of $\overline{\|E M S T\|}$ of the two groups which are calculated independently, the upper bound of the total $\overline{\|E M S T\|}$ can be represented as

$$
\overline{\|E M S T\|}<c(d) \sqrt{q_{i}} \int_{0}^{2 \pi} \int_{0}^{\frac{q^{\frac{\alpha}{2 \alpha-2}}}{\sqrt{n}}} x \sqrt{f_{n}^{(1)}(x, \theta)} d x d \theta+\Upsilon_{q_{i}},
$$

when $q_{i}=O\left(n^{\frac{\alpha-1}{\alpha}}\right)$. Moreover, the total $\overline{\|E M S T\|}$ must be greater than the $\overline{\|E M S T\|}$ of nodes within the range of $\frac{\sqrt{q_{i}}}{\sqrt{n}}$ from the center. Therefore, the $\overline{\|E M S T\|}$ is lower-bounded by

$$
\overline{\|E M S T\|}>c(d) \sqrt{q_{i}} \int_{0}^{2 \pi} \int_{0}^{\frac{\sqrt{q_{i}}}{\sqrt{n}}} \sqrt{\frac{x^{2} f_{n}(x, \theta)}{\int_{0}^{2 \pi} \int_{0}^{\frac{\sqrt{q_{i}}}{\sqrt{n}}} x f_{n}(x, \theta) d x d \theta}} d x d \theta .
$$

Consequently, according to 80 and 81 , the $\overline{\|E M S T\|}$ is summarized in (60). Moreover, since the capacity ratio of social networks and traditional ad hoc networks is greater than $\tilde{\Theta}(1)$ when $\alpha>1$, which is shown in Section VI, we use $\tilde{\Theta}(\cdot)$ instead of $\Theta(\cdot)$ here and ignore the poly-logarithmic factors.

Denoting the expectation of the minimum total number of hops for a multicast tree is $N_{q}(n)$. If the packet is transmitted through the path which is close to the line of multicast tree within $\Theta\left(\sqrt{\frac{\log n}{n}}\right)$, the order of the total length of the path equals to $\overline{\|E M S T\|}$ in order sense. However, another important case must be considered, which is that one node can transmit to multiple nodes simultaneously if the distance between them are smaller than $r(n)$. Thus, these hops can be treated as one, and we denote this as 'hop reduction'. For example, if there are $k_{0}$ destinations distributed in a small region with side length less than $r(n)$ uniformly and independently, the total length of the EMST among them is $r(n) \sqrt{k_{0}}$ which consists at least $k_{0}$ hops. But the total hop for them is 1 since the source can transmit to other nodes simultaneously. In our network model, the number of nodes in a region with side length $r(n)$ is $\Theta(\log n)$. Thus, the relation between $N_{q}(n)$ and $\overline{\|E M S T\|}$ is

$$
N_{q}(n)=O\left(\frac{\overline{\|E M S T\|}}{r(n)}\right), \text { and } N_{q}(n)=\Omega\left(\frac{\overline{\|E M S T\|}}{r(n) \log n}\right),
$$

due to the 'hop reduction'. To obtain the order of $N_{q}(n)$, we give the following lemma.

Lemma 6 The expectation of the minimum total number of hops for a multicast tree satisfies

$$
N_{q}(n)= \begin{cases}\Theta\left(\frac{\overline{\|E M S T\|}}{r(n)}\right) & q=o\left(\frac{n}{\log n \log \log n}\right), \\ \tilde{\Theta}\left(\frac{\|E M S T\|}{r(n)}\right) & q=\Omega\left(\frac{n}{\log n \log \log n}\right),\end{cases}
$$

where $\alpha=1$, and

$$
N_{q}(n)= \begin{cases}\Theta\left(\frac{\overline{\|E M S T\|}}{r(n)}\right) & q=o\left(\frac{n}{\log n}\right), \\ \tilde{\Theta}\left(\frac{\overline{\|E M S T\|}}{r(n)}\right) & q=\Omega\left(\frac{n}{\log n}\right),\end{cases}
$$

where $0 \leq \alpha<1$, and

$$
N_{q}(n)=\tilde{\Theta}\left(\overline{\frac{\|E M S T\|}{r(n)}}\right)
$$

where $\alpha>1$.

Proof: Firstly, we prove the lemma under the condition that $\alpha=1$. Considering a square region with side length $r(n)$, which is $k r(n)$ away from the source, when the total number of destinations is $q$, the expectation of the number of destinations in this region can be upper-bounded as

$$
D_{k}<q \int_{0}^{\frac{1}{k}} \int_{k}^{(k+1) \sqrt{\frac{\log n}{n}}} f_{n}(x, \theta) d x d \theta .
$$

If $k>\sqrt{\frac{q}{C_{1}(q, n) \log n}}, D_{k}$ satisfies

$$
D_{k}<2 q \int_{0}^{\frac{1}{k}} \int_{k \sqrt{\frac{\log n}{n}}}^{(k+1) \sqrt{\frac{\log n}{n}}} \frac{\overline{c_{15}}}{x C_{1}(q, n)} d x d \theta<\frac{2 \overline{c_{15}} q}{k^{2} C_{1}(q, n)}
$$

where $\overline{c_{15}}$ is constant according to Theorem 11. If $k<$ $\sqrt{\frac{q}{C_{1}(q, n) \log n}}, D_{k}$ can be derived as

$$
D_{k}<2 q \int_{0}^{\frac{1}{k}} \int_{k \sqrt{\frac{\log n}{n}}}^{(k+1) \sqrt{\frac{\log n}{n}}} \frac{n x}{q} d x d \theta<2 \log n .
$$

Notice that if there is $o(1)$ destination in one region with side length $r(n)$ in average, there is no 'hop reduction'. When $k>\sqrt{\frac{2 \overline{c_{15}} q}{C_{1}(q, n)}}$, which means $\frac{2 \overline{c_{15}} q_{i}}{k^{2} C_{1}(q, n)}<1$, there is no 'hop reduction'. Moreover, if $k<\sqrt{\frac{q}{C_{1}(q, n) \log n}}$, the number of 'hop reduction' is smaller than $\sqrt{\log n}$ in each region with side length $r(n)$. Finally, the total number of 'hop reduction' is the sum of the two parts, i.e., $k<\sqrt{\frac{q}{C_{1}(q, n) \log n}}$ and $\sqrt{\frac{q}{C_{1}(q, n) \log n}}<k<\min \left\{\sqrt{\frac{2 \overline{c_{15}} q}{C_{1}(q, n)}}, \frac{\sqrt{n}}{2 \sqrt{\log n}}\right\}$, where $\frac{\sqrt{n}}{2 \sqrt{\log n}}$ is the maximum value of $k$. Thus, according to 87 , 
the total number of 'hop reduction' is upper-bounded by

$$
\begin{gathered}
\quad \frac{\pi \sqrt{\log n} q}{C_{1}(q, n) \log n}+\sum_{k=\sqrt{\overline{C_{1}(q, n) \log n}}}^{\min \left\{\sqrt{\left.\frac{2 \overline{c_{15} q}}{C_{1}(q, n)}, \frac{\sqrt{n}}{2 \sqrt{\log n}}\right\}} \frac{4 \overline{c_{15}} \pi q}{k C_{1}(q, n)}\right.} \\
< \begin{cases}\frac{2 \overline{c_{15}} \pi q \log 2 \overline{c_{15}} \log n}{C_{1}(q, n)} & q \leq \frac{C_{1}(q, n) n}{8 \overline{c_{15}} \log n}, \\
\frac{2 \overline{c_{15}} \pi q \log \frac{n C_{1}(q, n)}{2 q}}{C_{1}(q, n)} & q>\frac{C_{1}(q, n) n}{8 \overline{c_{15}} \log n} .\end{cases}
\end{gathered}
$$

Furthermore, when $q<\frac{n}{\log n \log \log n}$, the total number of hops of the EMST is lower-bounded as

$$
\begin{aligned}
\frac{\overline{\|E M S T\|}}{r(n)} & >\frac{c_{10} c(d) \pi}{2} \sqrt{\frac{q n}{C_{1}(q, n) \log n}} \\
& >\frac{4 \overline{c_{15}} \pi q \log 2 \overline{c_{15}} \log n}{C_{1}(q, n)},
\end{aligned}
$$

when $n$ goes to infinite. Hence, the 'hop reduction' can be ignored in this condition in order sense. Consequently, according to 82 , the expectation of the minimum total number of hops for a multicast tree is as in 83 when $\alpha=1$.

Similarly, the expectation of the minimum total number of hops for the case $0 \leq \alpha<1$ and $\alpha>1$ can be derived, and the results are demonstrated in (84) and (85), respectively.

According to Lemma 6, the capacity bound of this social network model can be derived in the following theorem.

Theorem 3 The expectation of the capacity of this social network model is bounded by

$$
C(n)=\left\{\begin{array}{cl}
\tilde{\Theta}\left(\frac{1}{n}\right) & 0 \leq \beta \leq 1, \\
\tilde{\Theta}\left(n^{\beta-2}\right) & 1<\beta \leq \frac{3}{2}, \\
\Theta\left(\frac{1}{\sqrt{n \log n}}\right) & \beta>\frac{3}{2}
\end{array}\right.
$$

when $0 \leq \alpha<1$, and

$$
C(n)=\left\{\begin{array}{cl}
\tilde{\Theta}\left(n^{-1}\right) & 0 \leq \beta \leq 1, \\
\tilde{\Theta}\left(n^{\beta-2}\right) & 1<\beta \leq \frac{3}{2}, \\
\Omega\left(\frac{\log ^{\beta-\frac{3}{2}} n}{\sqrt{n}}\right) & \frac{3}{2}<\beta<2, \\
\Omega\left(\frac{\sqrt{\log n}}{\sqrt{n} \log \log n}\right) & \beta=2, \\
\Theta\left(\sqrt{\frac{\log n}{n}}\right) & \beta>2,
\end{array}\right.
$$

when $\alpha=1$, and

$$
C(n)=\tilde{\Theta}\left(\frac{1}{\mathbb{E}\{\overline{\|E M S T\|}\} \sqrt{n}}\right),
$$

when $\alpha>1$, and $\mathbb{E}\{\overline{\|E M S T\|}\}=\sum_{q=1}^{n} \frac{\overline{\|E M S T\|}}{q^{\beta} G_{n}}$. We do not show the detailed expressions for the sake of conciseness.

Proof: From Lemma 6, we know that the total number of hops is $N_{q}(n)$ for each multicast session. Therefore, the expectation of the total number of hops is $\mathbb{E}\left\{N_{q}(n)\right\}=$ $\sum_{q=1}^{n} \frac{N_{q}(n)}{q^{\beta} G_{n}}$. Furthermore, there are $n$ multicast sessions in the network. Since the transmission range is limited by $r(n)$, there are at most $\frac{1}{r^{2}(n)}$ active nodes in one time slot in average. Thus, the capacity of the network is bounded by

$$
\frac{1}{\mathbb{E}\left\{N_{q}(n)\right\} n r^{2}(n)}=\frac{1}{\mathbb{E}\left\{N_{q}(n)\right\} \log n} .
$$

The capacity bound can be calculated based on Theorem 2 and the results are presented in (91), (92) and (93).

\section{Simulations}

In this section, we will give simulations about the destination PDFs for the source which has $q$ destinations. In our simulations, the number of nodes $n$ is from $1 \times 10^{4}$ to $1 \times 10^{5}$, and the number of destinations is $q=n^{\frac{4}{5}}$. The distance range is $0 \leq x \leq 1$. The simulation results are averaged over 10000 realizations. For each realization, firstly, $n$ nodes are randomly and uniformly distributed in the network, and we assume that the source is at the center of the network. Afterwards, $q$ destinations are selected based on the rank-based model in 6. Finally, the statistical PDF of these nodes is obtained and compared with the theoretical result.

The statistical PDF is obtained according to the simulation results. On the other hand, the corresponding theoretical PDF is $f_{n}(x)=2 \pi x f_{n}(x, \theta)$, which can be expressed as

$$
f_{n}(x)=\left\{\begin{array}{cl}
\Theta\left(\frac{n^{\frac{1}{5}} x}{1+n^{\frac{1}{5}} x^{2 \alpha}}\right) & 0 \leq \alpha<1 \\
\Theta\left(\frac{n^{\frac{1}{5}} x}{1+n^{\frac{1}{5}} x^{2} \log n}\right) & \alpha=1 \\
\Theta\left(\frac{n^{\frac{1}{5}} x}{1+n^{\frac{\alpha}{5}} x^{2 \alpha}}\right) & \alpha>1
\end{array}\right.
$$

In our simulation, we use Surface Fitting to obtain the simulation results. Therefore, based on 95, we give the equation of surface fitting method as follows

$$
f_{n}(x)=\left\{\begin{array}{cl}
\frac{a_{1} n^{b_{1}} x^{c_{3}}}{1+d_{1} n^{e_{1}} x^{f_{1}}} & 0 \leq \alpha<1 \\
\frac{a_{2} n^{b_{2}} x^{c_{1}}}{1+d_{2} n^{e_{2}} x^{f_{2}} \log ^{g_{2}} n} & \alpha=1 \\
\frac{a_{3} n^{b_{3}} x^{c_{2}}}{1+d_{3} n^{e_{3}} x^{f_{3}}} & \alpha>1
\end{array}\right.
$$

where $a_{i}$ to $g_{i}$ are the surface fitting parameters. In our simulations, we only focus on $b_{i}, c_{i}, e_{i}, f_{i}$ and $g_{i}$ to show the order of the PDF.

The simulation results are illustrated in Figure 1 for the case $0 \leq \alpha<1$, and $\alpha$ is assumed to be 0.5 here. Moreover,

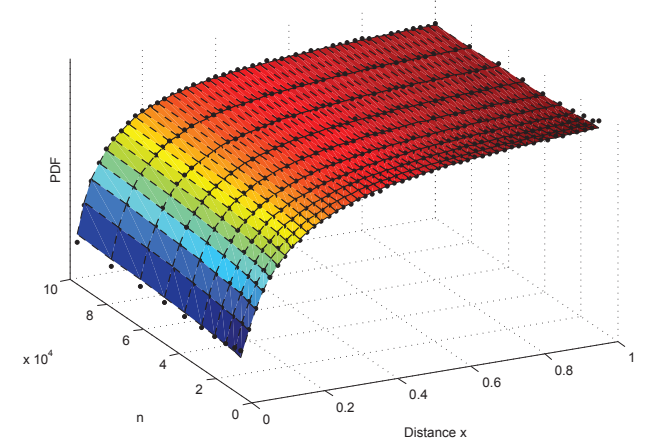

Fig. 1: The comparison of theoretical PDF and statistical PDF for $\alpha=0.5$.

the corresponding parameters are given in Table II. R-square in Table II is the coefficient of determination. If R-square is close to 1 , it means that the fitting is accurate. From the simulation 
TABLE II: Surface fitting parameters

\begin{tabular}{|c|c|c|c|}
\hline Parameters & $\alpha=0.5$ & $\alpha=1$ & $\alpha=1.2$ \\
\hline$a_{i}$ & 2.841 & 2.164 & 2.421 \\
\hline$b_{i}$ & 0.1886 & 0.2002 & 0.1948 \\
\hline$c_{i}$ & 1.07 & 1.057 & 1.075 \\
\hline$d_{i}$ & 1.658 & 0.5765 & 2.109 \\
\hline$e_{i}$ & 0.2098 & 0.1983 & 0.2546 \\
\hline$f_{i}$ & 1.066 & 2.056 & 2.473 \\
\hline$g_{i}$ & - & 0.8885 & - \\
\hline$R-$ square & 0.9996 & 0.9998 & 0.9992 \\
\hline
\end{tabular}

results we can find that the differences between the parameters of surface fitting function and theoretical function are no more than $7 \%$, which indicates that our theoretical PDF is very close to the simulation one in order sense.

We also give the simulations for the case $\alpha=1$ and $\alpha>1$ in Figure 2 and 3, respectively. The corresponding parameters are listed in Table II. For the case $\alpha>1$, we assume $\alpha=1.2$.

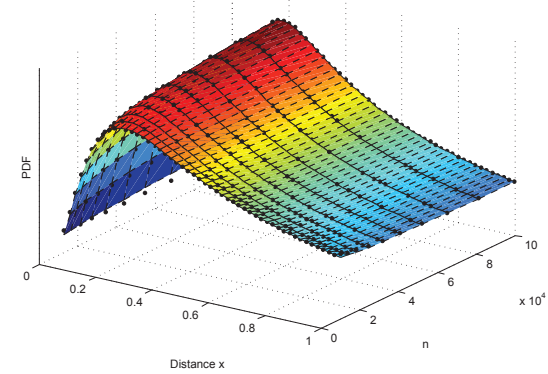

Fig. 2: The comparison of theoretical PDF and statistical PDF for $\alpha=1$.

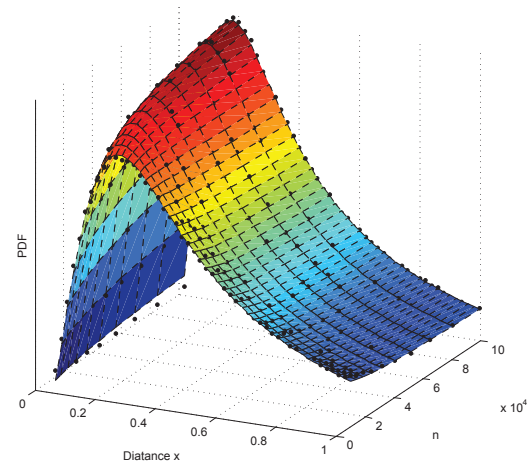

Fig. 3: The comparison of theoretical PDF and statistical PDF for $\alpha=1.2$.

From Figure 2 and 3, we can find that the theoretical PDFs are very close to the simulation ones in order sense. In Table II, when $\alpha=1$, the differences between the parameters of surface fitting function and theoretical function are no more than $5.7 \%$ except for $g_{2}$. The difference of $g_{2}$ is $11.2 \%$ because it is the power of $\log n$, which is not very large even when $n=1 \times 10^{5}$. Therefore, the surface fitting parameter $g_{2}$ is not accurate enough. However, this difference will not seriously influence the PDF because $\log n$ is quite small comparing with $n$ when $n$ is large enough. For the case $\alpha=1.2$, the differences between the parameters of surface fitting function and theoretical function are no more than $7.5 \%$. Therefore, from Figure 1-3 and Table II, it is indicated that the theoretical PDF can describe the real PDF of destinations in order sense for all cases of $\alpha$.

\section{Capacity Achieving Scheme}

A social network transmission scheme will be proposed in this section, and we will analyze the corresponding throughput. In this scheme, TDMA medium access control scheme based on the protocol model is employed. The network is divided into many cells with side-length $R_{0} r(n)$ where $R_{0}$ is constant. Therefore, $M^{2}$-TDMA can be employed, which allows each $M^{2}$ adjacent cells to be active with a round-robin fashion, where $M$ is constant. Moreover, considering the protocol model, $M$ must satisfy $M R_{0} r(n) \geq(1+\Delta) r(n)$, i.e., $M \geq \frac{1+\Delta}{R_{0}}$.

The EMST is established for the social networks and the EMST-based scheme is as follows.

1) Initially, the network is separated into cells, and TDMA is employed as described above.

2) For each arbitrary source $i$, an EMST is built among the $q_{i}$ destinations and node $i$. This tree is the basic EMST.

3) Considering the limited transmission range $r(n)$, each direct link in basic EMST can be constructed by sending packets hop by hop along the cells where link lines across. When a node is allowed to be active in this time slot, it will select the oldest packet in its buffer and transmit it to the next node according to the route of EMST.

To analyze the throughput of the EMST-based scheme, we propose the following theorem.

Theorem 4 The throughput of EMST-based scheme which is proposed above is of the same order as the capacity given in Theorem 3 when $0 \leq \alpha<2$ or $0 \leq \beta \leq 1$. Moreover, for the case $\alpha \geq 2$ and $\beta>1$, there is a gap $n^{\eta}$ between them, where $\eta>0$ is an arbitrary small constant.

Proof: We define the node set $S_{i}$ consisting the nodes which are one of the relays or destinations for one multicast session with source $i$. Considering an arbitrary cell $s$, the number of multicast sessions that invoke $s$ can be represented as

$$
N(s)=\sum_{i=1}^{n} \mathbf{1}_{\left\{s \text { is invoked by } S_{i}\right\}},
$$

where the $\mathbf{1}_{\left\{s \text { is invoked by } S_{i}\right\}}$ are i.i.d. Bernoullian random variables. Thus, according to the definition of throughput, this theorem is equivalent to

$$
\operatorname{Pr}\left\{\bigcap_{s}\{N(s)<c n \overline{\|E M S T\|} r(n)\}\right\} \rightarrow 1,
$$

when $0 \leq \alpha<2$, and

$$
\operatorname{Pr}\left\{\bigcap_{s}\left\{N(s)<c n^{1+\eta} \overline{\|E M S T\|} r(n)\right\}\right\} \rightarrow 1,
$$

when $\alpha \geq 2$ and $c>0$ is constant. 
In order to prove 98 and 99 , we consider a region which is defined as

$$
\begin{gathered}
R_{i}=\{x \mid \text { The minimum distance from } x \text { to the } \\
\left.E M S T_{i} \text { is smaller than } 2 R_{0} r(n)\right\} .
\end{gathered}
$$

Therefore, all the invoked cells by $S_{i}$ is covered by $R_{i}$, and its average size is no greater than $3 R_{0} \overline{\left\|E M S T_{i}\right\|} r(n)$. Since the $s$ is randomly selected, mean value of $\mathbf{1}_{\left\{s \text { is invoked by } S_{i}\right\}}$ is $m_{1}^{(i)}<\min \left\{3 R_{0} \overline{\left\|E M S T_{i}\right\|} r(n), 1\right\}=m_{2}^{(i)}$.

Since there are total $n$ EMSTs, $N(s)$ is upper-bounded by $n$. Thus, throughput is lower-bounded by $\frac{1}{n}$, which indicates that the capacity bound in Theorem 4 can be achieved when $0 \leq \beta \leq 1$.

For the case $\beta>1, q$ represents the mean of $q_{i}$, which satisfies $q=\Theta\left(\max \left\{n^{2-\beta}, 1\right\}\right)$. Firstly, we prove the theorem for the case $0<\alpha<1$. Considering two multicast sessions with source $i$ and $j$, the sets of their friends are defined as $F_{i}$ and $F_{j}$, respectively. Therefore, the correlation between $\mathbf{1}_{\left\{s \text { is invoked by } S_{i}\right\}}$ and $\mathbf{1}_{\left\{s \text { is invoked by } S_{j}\right\}}$ is related with the total length of links among nodes in set $F_{i} \cup F_{j}$. For the case $0<$ $\alpha<1$, the number of nodes $k$ in $F_{i} \cup F_{j}$ can be upper-bounded as

$$
\begin{aligned}
\left|F_{i} \cup F_{j}\right| & <\sum_{X=1}^{n} q^{2} \mathbb{P}\left\{\operatorname{Rank}_{i}(k)=X\right\} \mathbb{P}\left\{\operatorname{Rank}_{j}(k)=X\right\} \\
& <\overline{c_{16}} q\left(\frac{q}{n}\right)^{\frac{1-\alpha}{\alpha}}=o(q),
\end{aligned}
$$

where $\overline{c_{16}}$ is constant according to Lemma 5 Furthermore, the probability that a node belongs to $F_{i} \cup F_{j}$ is small if it is far from $i$ and $j$ due to Theorem 11. However, the links connected to it in the $E M S T_{i}$ and $E M S T_{j}$ are long. Thus, the total length of links among nodes in set $F_{i} \cup F_{j}$ is smaller than $\overline{\left\|E M S T_{i}\right\|}$ and $\overline{\left\|E M S T_{j}\right\|}$ in order sense. Hence, the correlation between $\mathbf{1}_{\left\{s \text { is invoked by } S_{i}\right\}}$ and $\mathbf{1}_{\left\{s \text { is invoked by } S_{j}\right\}}$ can be ignored. Therefore, denoting $N(s)$ as another sum of i.i.d. Bernoullian random variables with mean $m_{2}^{(i)}$, we can find that $\hat{N}(s)$ is statistically larger than $N(s)$. Thus, based on Chernoff bounds, we can obtain the following relation

$$
\begin{aligned}
\operatorname{Pr}\{N(s)>2 \mathbb{E}[\hat{N}(s)]\} & <\operatorname{Pr}\{\hat{N}(s)>2 \mathbb{E}[\hat{N}(s)]\} \\
& <(e)^{-n m_{2} / 3}
\end{aligned}
$$

where $m_{2}$ is the mean value of $m_{2}^{(i)}$ which equals to $\min \left\{3 R_{0} \overline{\|E M S T\|} r(n), 1\right\}$. Then we consider all the cells, since $m_{2}>3 R_{0} r(n)$,

$$
\begin{aligned}
& \operatorname{Pr}\left\{\bigcap_{s}\left\{N(s)<6 R_{0} n \overline{\|E M S T\|} r(n)\right\}\right\} \\
\geq & 1-\sum_{s} \operatorname{Pr}\{N(s)>2 \mathbb{E}[\hat{N}(s)]\} \\
\geq & 1-n e^{-3 R_{0} \sqrt{n \log n} / 3} \rightarrow 1 \text { as } n \rightarrow \infty .
\end{aligned}
$$

Therefore, the throughput lower-bound of the proposed scheme is $\Theta\left(\frac{1}{n \overline{\|E M S T\|} r(n)}\right)$ based on the definition of throughput. Moreover, considering the capacity upper-bound in Theorem 3. which is the same as the lower-bound in order sense, the proposed scheme achieves the capacity upper-bound.

For the case $\alpha=1$, the number of nodes in $F_{i} \cup F_{j}$ can be upper-bounded as

$$
\begin{aligned}
\left|F_{i} \cup F_{j}\right| & <\sum_{X=1}^{n} q^{2} \mathbb{P}\left\{\operatorname{Rank}_{i}(k)=X\right\} \mathbb{P}\left\{\operatorname{Rank}_{i}(k)=X\right\} \\
& <\frac{\overline{c_{17}} q}{C_{1}(q, n)}=o(q),
\end{aligned}
$$

where $\overline{c_{17}}$ is constant according to Lemma 5 Therefore, it is similar to the case $0<\alpha<1$ that the correlation between $\mathbf{1}_{\left\{s \text { is invoked by } S_{i}\right\}}$ and $\mathbf{1}_{\left\{s \text { is invoked by } S_{j}\right\}}$ can be ignored, and the proposed scheme can be proved to achieve the capacity upper-bound in order sense.

For the case $\alpha>1$, we consider two sources $i$ and $j$, and the distance between them satisfies $d(i, j)>q^{\frac{1-2 \varepsilon}{2}} n^{\frac{2 \varepsilon-1}{2}}$, where $0<\varepsilon<1 / 3$ is a small constant. Then, the nodes in the network can be divided into 3 parts.

- Part 1: Defined as set $P 1=\left\{k \mid \operatorname{Rank}_{i}(k)<q^{1-\varepsilon} n^{\varepsilon}\right\}$.

- Part 2: Defined as set $P 2=\left\{k \mid \operatorname{Rank}_{j}(k)<q^{1-\varepsilon} n^{\varepsilon}\right\}$.

- Part 3: Defined as set $P 3=\left\{k \mid \operatorname{Rank}_{i}(k)>\right.$ $q^{1-\varepsilon} n^{\varepsilon}$ and $\left.\operatorname{Rank}_{j}(k)>q^{1-\varepsilon} n^{\varepsilon}\right\}$.

Since the nodes are uniformly and randomly distributed in the network, it is obvious that $\operatorname{Rank}_{i}(k)>q^{1-\varepsilon} n^{\varepsilon}$ when $k \in$ $P 1$ and $\operatorname{Rank}_{j}(k)>q^{1-\varepsilon} n^{\varepsilon}$ when $k \in P 2$ with probability 1. Therefore, the number of nodes in $F_{i} \cup F_{j}$ can be upperbounded by

$$
\begin{aligned}
& \left|F_{i} \cup F_{j}\right| \\
= & \left|F_{i} \cup F_{j} \cup P 1\right|+\left|F_{i} \cup F_{j} \cup P 2\right|+\left|F_{i} \cup F_{j} \cup P 3\right| \\
< & \sum_{X=1}^{q^{1-\varepsilon} n^{\varepsilon}} q^{2} \mathbb{P}\left\{\operatorname{Rank}_{i}(k)=X\right\} \mathbb{P}\left\{\operatorname{Rank}_{j}(k)=q^{1-\varepsilon} n^{\varepsilon}\right\} \\
& +\sum_{X=1}^{q^{1-\varepsilon} n^{\varepsilon}} q^{2} \mathbb{P}\left\{\operatorname{Rank}_{i}(k)=q^{1-\varepsilon} n^{\varepsilon}\right\} \mathbb{P}\left\{\operatorname{Rank}_{j}(k)=X\right\} \\
& +\sum_{X=q^{1-\varepsilon} n^{\varepsilon}+1}^{n} q^{2} \mathbb{P}\left\{\operatorname{Rank}_{i}(k)=X\right\} \mathbb{P}\left\{\operatorname{Rank}_{j}(k)=X\right\} \\
< & \overline{c_{18}} q\left(\frac{q}{n}\right)^{\varepsilon \alpha}+\overline{c_{19}} q\left(\frac{q}{n}\right)^{\varepsilon \alpha}+\overline{c_{20}} q\left(\frac{q}{n}\right)^{(2 \alpha-1) \varepsilon} \\
= & o(q),
\end{aligned}
$$

where $\overline{c_{18}}, \overline{c_{19}}, \overline{c_{20}}$ are constant according to Lemma 5

Therefore, the correlation between $\mathbf{1}_{\left\{s \text { is invoked by } S_{i}\right\}}$ and $\mathbf{1}_{\left\{s \text { is invoked by } S_{j}\right\}}$ can be ignored if $d(i, j)>q^{\frac{1-2 \varepsilon}{2}} n^{\frac{2 \varepsilon-1}{2}}$. According to this result, we divide all of the nodes into $q^{1-3 \varepsilon} n^{3 \varepsilon}$ groups with the same nodes number, and the distance between nodes in the same group is larger than $q^{\frac{1-2 \varepsilon}{2}} n^{\frac{2 \varepsilon-1}{2}}$. Owing to the uniform and independent distribution of nodes, this division exists with probability 1 when $n$ goes to infinity. Hence, we define $G_{u}$ as $G_{u}=\sum_{i \text { is in group } u} \mathbf{1}_{\left\{s \text { is invoked by } S_{i}\right\}}$ and $\hat{G}_{u}$ as the corresponding sum of i.i.d. Bernoullian random variables with mean $m_{3}^{(i)}=\min \left\{3 R_{0} n^{\eta} \overline{\left\|E M S T_{i}\right\|} r(n), 1\right\}$, where $\eta \geq 0$ is a small constant.

Considering the case that there are some links belonging to both $E M S T_{i}$ and $E M S T_{j}, s$ is more likely to be invoked by $S_{j}$ if it is invoked by $S_{i}$. On the other hand, if the common links of $E M S T_{i}$ and $E M S T_{j}$ do not invoke $s$, $\mathbf{1}_{\left\{s \text { is invoked by } S_{i}\right\}}$ and $\mathbf{1}_{\left\{s \text { is invoked by } S_{j}\right\}}$ are independent from each other. Therefore, the correlation coefficient between 
$\mathbf{1}_{\left\{s \text { is invoked by } S_{i}\right\}}$ and $\mathbf{1}_{\left\{s \text { is invoked by } S_{j}\right\}}$ is non-negative. Thus, the correlation coefficients between different $G_{u}$ are also nonnegative. Consequently, we can obtain the following relation

$$
\begin{aligned}
\operatorname{Pr}\{N(s)>2 \mathbb{E}[\hat{N}(s)]\} & =\operatorname{Pr}\left\{G_{u}>2 \mathbb{E}[\hat{N}(s)]\right\} \\
& <\operatorname{Pr}\left\{\max _{u}\left\{G_{u}\right\}>\frac{2 \mathbb{E}[\hat{N}(s)]}{q^{1-3 \varepsilon} n^{3 \varepsilon}}\right\} \\
& =1-\operatorname{Pr}\left\{\bigcap_{u} G_{u}<\frac{2 \mathbb{E}[\hat{N}(s)]}{q^{1-3 \varepsilon} n^{3 \varepsilon}}\right\} \\
& <\sum_{u} \operatorname{Pr}\left\{G_{u}<\frac{2 \mathbb{E}[\hat{N}(s)]}{q^{1-3 \varepsilon} n^{3 \varepsilon}}\right\} \\
& <q^{1-3 \varepsilon} n^{3 \varepsilon}(e)^{-\left(\frac{n}{q}\right)^{1-3 \varepsilon} m_{3} / 3},
\end{aligned}
$$

According to 81,

$$
m_{3} \geq \min \left\{3 R_{0} \sqrt{\log n}\left(\frac{q}{n}\right)^{\frac{\min \{\alpha, 2\}}{2}} n^{\frac{\eta}{2}}, 1\right\},
$$

and therefore

$$
\begin{aligned}
& \operatorname{Pr}\left\{\bigcap_{s}\left\{N(s)<6 R_{0} n^{1+\eta} \overline{\|E M S T\|} r(n)\right\}\right\} \\
\geq & 1-\sum_{s} \operatorname{Pr}\{N(s)>2 \mathbb{E}[\hat{N}(s)]\} \\
\geq & 1-q^{1-3 \varepsilon} n^{1+3 \varepsilon} e^{-\left(\frac{n}{q}\right)^{1-3 \varepsilon} \min \left\{3 R_{0} \sqrt{\log n}\left(\frac{q}{n}\right)^{\frac{\min \{\alpha, 2\}}{2}} n^{\frac{\eta}{2}}, 1\right\} / 3} \\
= & 1-q^{1-3 \varepsilon} n^{1+3 \varepsilon} e^{-n^{c_{\beta}(1-3 \varepsilon)} \min \left\{3 R_{0} \sqrt{\log n} n^{c_{\beta} c_{\alpha}} n^{\frac{\eta}{2}}, 1\right\} / 3},
\end{aligned}
$$

where $\beta>1, c_{\beta}=\min \{\beta-1,1\}$ and $c_{\alpha}=\frac{\min \{\alpha, 2\}}{2}$. We select $\varepsilon=\frac{2-\alpha}{12}$ and $\eta=0$ when $1<\alpha<2$, and $\varepsilon=\frac{\eta}{12}$ and $\eta>0$ when $\alpha \geq 2$, and therefore the value of 108 becomes 1 when $n$ goes infinity. Thus, the proposed scheme achieves the capacity upper-bound when $1<\alpha<2$, and there is a gap $n^{\eta}$ between them when $\alpha \geq 2$. Consequently, the theorem is proved.

\section{Discussions}

In this session, we discuss about the difference of capacity between our social networks and traditional multicast ad hoc networks. The authors in [2] show that the capacity of the networks with uniformly and randomly selected destinations is $C_{\text {traditional }}(n)=\Theta\left(\frac{1}{N_{q}(n) n r^{2}(n)}\right)$, where $N_{q}(n)$ is the average total number of hops of a multicast session, and $r(n)$ is the transmission range. Therefore, the difference of capacity is only caused by the difference of $N_{q}(n)$. Moreover, [2] shows that

$$
N_{q}(n)= \begin{cases}\Theta\left(\frac{\overline{\|E M S T\|}}{r(n)}\right) & q=o\left(r^{-2}(n)\right), \\ \Theta\left(r^{-2}(n)\right) & q=\Omega\left(r^{-2}(n)\right),\end{cases}
$$

where $q$ is the number of destinations. The similar results can be found in [3] and [4]. Since the number of destinations is certain before transmission in their networks, we compare the capacity of our network with theirs under the assumption that the number of destinations is certain. The capacity ratio is defined as

$$
R_{q}=\frac{C_{\text {social }}^{(q)}(n)}{C_{\text {traditional }}^{(q)}(n)},
$$

where $C_{\text {social }}^{(q)}(n)$ is the capacity of social networks when destination number is $q$, and $C_{\text {traditional }}^{(q)}(n)$ is the corresponding capacity for traditional ad hoc networks in [2]- [4].

Comparison for $0 \leq \alpha<1$. In this case, $\overline{\|E M S T\|}=\Theta(\sqrt{q})$ in both of our networks 59 and traditional multicast ad hoc networks [2]. Therefore, the capacity ratio is

$$
R_{q}= \begin{cases}\Theta(1) & q=O\left(\frac{n}{\log n}\right), \\ \tilde{\Theta}(1) & q=\omega\left(\frac{n}{\log n}\right) .\end{cases}
$$

From the expression of PDF of friends in Theorem 1 it can be found that the concentration degree of friends is determined by $\alpha$. In this case, the value of $\alpha$ is small, and thus the PDF is similar to the uniform distribution in order sense. Therefore, the order of $\overline{\|E M S T\|}$ in social network is the same as the traditional ad hoc networks.

Comparison when $\alpha=1$. In this case, $\overline{\|E M S T\|}=\Theta(\sqrt{q})$ in traditional multicast ad hoc networks. However, in our social networks, according to Theorem 2, the $\overline{\|E M S T\|}$ is smaller. Thus, the capacity ratio is

$$
R_{q}= \begin{cases}\Omega\left(\frac{\log n}{\sqrt{q}}\right) & q=O(\log n), \\ \Theta(\sqrt{\log n}) & q=\omega(\log n), q=o\left(\frac{n}{\log n \log \log n}\right), \\ \tilde{\Theta}(1) & q=\Omega\left(\frac{n}{\log n \log \log n}\right) .\end{cases}
$$

When $\alpha=1$, the majority of nodes are centralized within a small region close to the source. Due to the concentration, the average distance between nodes is decreased comparing with the uniform distribution. Furthermore, the $\overline{\|E M S T\|}$ is decreased, and the capacity is increased.

Comparison for $\alpha>1$. In this case, we use $\tilde{\Theta}(\cdot)$ instead of $\Theta(\cdot)$ to make the result brief. Firstly, $\overline{\|E M S T\|}=\Theta(\sqrt{q})$ in traditional multicast ad hoc networks. Based on the corresponding $\overline{\|E M S T\|}$ in 60 , the capacity ratio is

$$
R_{q}= \begin{cases}\Omega\left(q^{\frac{1}{2}-\alpha} n^{\alpha-1}\right) & q_{i}=O\left(n^{\frac{\alpha-1}{\alpha}}\right), 1<\alpha \leq \frac{3}{2}, \\ \tilde{\Theta}\left(\left(\frac{n}{q}\right)^{\frac{\alpha-1}{2}}\right) & q_{i}=\omega\left(n^{\frac{\alpha-1}{\alpha}}\right), 1<\alpha \leq \frac{3}{2}, \\ \Omega\left(q^{\frac{1}{2-2 \alpha}} \sqrt{n}\right) & q_{i}=O\left(n^{\frac{\alpha-1}{\alpha}}\right), \frac{3}{2}<\alpha<2, \\ \tilde{\Theta}\left(\left(\frac{n}{q}\right)^{\frac{\alpha-1}{2}}\right) & q_{i}=\omega\left(n^{\frac{\alpha-1}{\alpha}}\right), \frac{3}{2}<\alpha<2, \\ \tilde{\Theta}\left(\sqrt{\frac{n}{q}}\right) & \alpha \geq 2 .\end{cases}
$$

The capacity ratio is larger than other two cases because that the $\alpha$ is sufficiently large and the destinations are centralized. Thus, most of the destinations are distributed around the source, which causes large $R_{q}$.

Figure 4 illustrates the variances of $R_{q}$ versus $\alpha$ when $q=\Theta\left(n^{\frac{2}{3}}\right)$ in order sense. In Figure 4, when $\alpha=0$, the network is the traditional ad hoc network. When $0<\alpha<1$, the distribution of destination is similar to uniformly distribution, and therefore the corresponding capacity ratio is $\Theta(1)$. When $\alpha=1$, the destinations distribute closer to the source than that of case $0 \leq \alpha<1$, which results in a smaller $\overline{\|E M S T\|}$. Therefore, the capacity ratio becomes $\Theta(\log n)$ which is greater than $\Theta(1)$. Moreover, when $1<\alpha<2$, the concentration degree of destinations becomes higher when $\alpha$ is larger. Hence, the capacity ratio increases with $\alpha$. However, 
when $\alpha \geq 2$, since the density of destinations is limited by the node density of all the $n$ nodes in the network, the concentration degree of destinations does not increase with $\alpha$ in this case. Thus, the corresponding capacity ratio does not change with $\alpha$ here. In Figure 4, we ignore poly-logarithmic factors when $\alpha>1$.

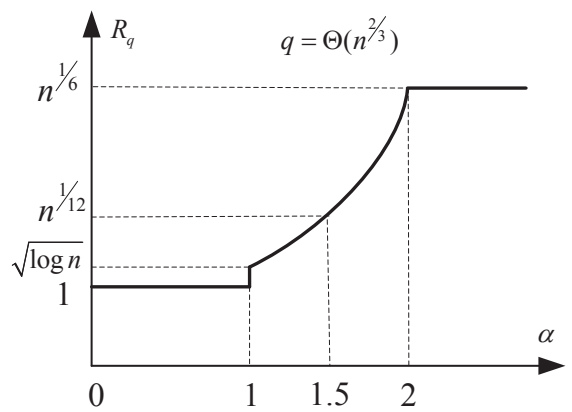

Fig. 4: The variances of $R_{q}$ versus $\alpha$. When $\alpha=0$, the network is the traditional ad hoc network

\section{CONCLUSION}

In this paper, we introduce the two-layer network model, which includes social layer and networking layer. In the social layer, the social group size of each source node is modeled as power-law distribution model. Moreover, the rank-based model is utilized to describe the relation between source and destinations in the networking layer. In this model, the PDF of destinations is derived and verified by numerical simulations. Based on the PDF, we analyze the capacity bound of the networks and propose an EMST-based scheme to achieve the capacity in most cases. Finally, the social network capacity is compared with the traditional ad hoc networks.

\section{ACKNOWLEDGEMENT}

This work is supported by NSF China (No.61325012, 61271219, 61221001, 61428205);China Ministry of Education Doctor Program(No.20130073110025); Shanghai Basic Research Key Project (12JC1405200, 11JC1405100);Shanghai International Cooperation Project: (No. 13510711300).

\section{APPENDIX}

We prove the Lemma 2 based on the following lemma. The proof of Lemma 7 for the case $f_{n}(\mathbf{x})$ related with $n$ is similar to the proof of Theorem 3 in [21], and we do not show it here due to the space limitation.

Lemma 7 In the network with size $[0,1]^{d}$, we denote the EMSTs of nodes with distribution $f_{n}(\mathbf{x})$ and $g_{n}(\mathbf{x})$ as $E M S T_{f}$ and $E M S T_{g}$, respectively, where $f_{n}(\mathbf{x})$ is a continuous distribution and $g_{n}(\mathbf{x})$ is a blocked distribution. The total length of $E M S T_{f}$ can be expressed as

$$
\lim _{n \rightarrow \infty} \frac{\overline{\left\|E M S T_{f}\right\|} n^{-\frac{d-1}{d}}}{\int_{R^{d}} f_{n}(\mathbf{x})^{\frac{d-1}{d}} d \mathbf{x}}=c(d)
$$

when the following two requirements are satisfied, where $c(d)$ is a constant.
- Requirement 1: The total lengths of $E M S T_{f}$ and $E M A T_{g}$ satisfy

$$
\lim _{n \rightarrow \infty} \frac{\left|\overline{\left\|E M S T_{f}\right\|}-\overline{\left\|E M S T_{g}\right\|}\right|}{\left|\left\{i: \mathbf{x}_{i}^{f} \neq \mathbf{x}_{i}^{g}\right\}\right|^{\frac{d-1}{d}}} \leq B
$$

where $B$ is a constant, $\left\{\mathbf{x}_{i}^{f}\right\}$ and $\left\{\mathbf{x}_{i}^{g}\right\}$ are the sets of the node positions of EMST and $E M A T_{g}$, respectively.

- Requirement 2: The total length of EMST can be expressed as

$$
\lim _{n \rightarrow \infty} \frac{\overline{\left\|E M S T_{g}\right\|} n^{\frac{1-d}{d}}}{\int_{R^{d}} g_{n}(\mathbf{x})^{\frac{d-1}{d}} d \mathbf{x}}=c(d) .
$$

The proof of Lemma 2.5 in [21] can be used to prove that the Requirement 1 in Lemma 7 is satisfied when the Condition 1 in Lemma 2 holds, and we do not show it here due to the space limitation. Therefore, it only remains to prove that Requirement 2 is also satisfied when the two conditions of Lemma 2 hold.

Firstly, we calculate the upper-bound of $\overline{\left\|E M S T_{g}\right\|}$. Considering a $d$-dimensional hypercube network with size $S$ (side length $S^{\frac{1}{d}}$ ), it has been proved in [21] that if there are $n$ nodes uniformly distributed in it, the total length of the corresponding EMST is $c_{0}(d) n^{\frac{d-1}{d}} S^{\frac{1}{d}}$ with probability 1 when $n$ goes to infinity, where $c_{0}(d)$ is a constant. Hence, defining

$$
\mathcal{X}=\left\{i: n \gamma_{i}\left|\xi_{i}\right|=\Theta(1)\right\}, \mathcal{Y}=\left\{i: n \gamma_{i}\left|\xi_{i}\right|=\omega(1)\right\},
$$

the upper-bound of $\overline{\left\|E M S T_{g}\right\|}$ can be expressed as

$\limsup _{n \rightarrow \infty} \frac{\overline{\left\|E M S T_{g}\right\|}}{M+c_{0}(d) \sum_{i \in \mathcal{Y}}\left(n \gamma_{i}\left|\xi_{i}\right|\right)^{\frac{d-1}{d}}\left|\xi_{i}\right|^{\frac{1}{d}}+\sum_{i \in \mathcal{X}} n \gamma_{i}\left|\xi_{i}\right| \sqrt{d}\left|\xi_{i}\right|^{\frac{1}{d}}} \leq 1$

where $\left|\xi_{i}\right|$ is the size of $\xi_{i}, M$ is the cost needed to unite all of the parts $\xi_{i}, c_{0}(d) \sum_{i \in \mathcal{Y}}\left(n \gamma_{i}\left|\xi_{i}\right|\right)^{\frac{d-1}{d}}\left|\xi_{i}\right|^{\frac{1}{d}}$ is the total length of EMST of the nodes in each part which belonging to $\mathcal{Y}$, and $\sum_{i \in \mathcal{X}} n \gamma_{i}\left|\xi_{i}\right| \sqrt{d}\left|\xi_{i}\right|^{\frac{1}{d}}$ is the upper-bound of it for $\mathcal{X}$. When two adjacent parts $\xi_{i}$ and $\xi_{j}$ are connected, the length of edge between them is no greater than $\sqrt{d}\left(\left|\xi_{i}\right|^{\frac{1}{d}}+\left|\xi_{j}\right|^{\frac{1}{d}}\right)$. Since there are at most $\zeta$ adjacent parts for each $\xi_{i}$, the $M$ in 118 satisfies $M \leq \zeta \sqrt{d} \sum_{i \in \mathcal{X} \cup \mathcal{Y}}\left|\xi_{i}\right|^{\frac{1}{d}}$ when each part only connects to its adjacent parts. Defining a constant $\varpi=\max _{i \in \mathcal{X}}\left\{\frac{\sqrt{d}\left(n \gamma_{i}\left|\xi_{i}\right|+\zeta\right)}{c_{0}(d)\left(n \gamma_{i}\left|\xi_{i}\right|\right)^{\frac{d-1}{d}}}\right\}$, we can obtain

$$
\lim _{n \rightarrow \infty} \frac{\overline{\left\|E M S T_{g}\right\|}}{\sum_{i \in \mathcal{X} \cup \mathcal{Y}}\left(n \gamma_{i}\left|\xi_{i}\right|\right)^{\frac{d-1}{d}}\left|\xi_{i}\right|^{\frac{1}{d}}} \leq \max \{2, \varpi\} c_{0}(d)
$$

Afterwards, we analyze the lower-bound of $\overline{\left\|E M S T_{g}\right\|}$. Let $D_{i}$ denote the set of edges $e$ in $E M S T_{g}$ such that both two endpoints of $e$ are in $\xi_{i}$. Let $V_{i}$ denote the set of nodes in $\xi_{i}$ which are jointed by an edge in $E M S T_{g}$ with one another endpoint out of $\xi_{i}$. Thus, the edges in $D_{i}$ and the EMST of $V_{i}$ connect all the nodes in $\xi_{i}$. Based on the Lemma 2.2 in [21], 
for any $i \in \mathcal{Y}$, the following relation holds when $n \rightarrow \infty$

$$
\frac{c_{0}(d)\left(n \gamma_{i}\left|\xi_{i}\right|\right)^{\frac{d-1}{d}}\left|\xi_{i}\right|^{\frac{1}{d}}}{\sum_{e \in d_{i}}|e|+c_{0}(d)\left(\left|V_{i}\right|\right)^{\frac{d-1}{d}}\left|\xi_{i}\right|^{\frac{1}{d}}} \leq 1
$$

where $\left|V_{i}\right|$ is the number of nodes in $V_{i},|e|$ is the length of $e, c_{0}(d)\left(n \gamma_{i}\left|\xi_{i}\right|\right)^{\frac{d-1}{d}}\left|\xi_{i}\right|^{\frac{1}{d}}$ is the total length of EMST among nodes in $\xi_{i}, \sum_{e \in d_{i}}|e|$ is the total length of edges with two endpoints in $\xi_{i}$, and the total length of EMST of the nodes connecting with the nodes outside $\xi_{i}$ can be bounded by $c_{0}(d)\left(\left|V_{i}\right|\right)^{\frac{d-1}{d}}\left|\xi_{i}\right|^{\frac{1}{d}}$.

In order to bound $\left|V_{i}\right|$, we consider a $d$-dimensional hypercube $\xi_{i}^{\prime}$ with side length $l_{i}=\frac{1}{2}\left|\xi_{i}\right|^{\frac{1}{d}}$ centered at the center point of $\xi_{i}$. For any node $v^{\prime}$ within $\xi_{i}^{\prime}$, if $v^{\prime} \in V_{i}$, we assume that it is connected with a node $v$ out of $\xi_{i}$ by an edge $e_{v^{\prime}} v$, which is denoted as the replaceable edge. Since $\xi_{i}^{\prime}$ is within $\xi_{i}$ and there are $\omega(1)$ nodes in $\xi_{i}-\xi_{i}^{\prime}$ (since $i \in \mathcal{Y}$ ), an appropriate $v^{*}$ satisfying $e_{v^{*} v}<e_{v^{\prime} v}$ can be found as a relay with probability 1 . Therefore $e_{v^{\prime} v}$ can be replaced by $e_{v^{\prime} v^{*}}+e_{v^{*} v}$, and thus $e_{v^{\prime} v^{*}}+e_{v^{*} v}<3 e_{v^{\prime} v^{\prime}}$. After replacing all the replaceable edges of each part $(i \in \mathcal{Y})$, the $E M S T_{g}$ becomes a new graph $G$. It should be noticed that there may be some circuits after replacing edges, and thus we denote it as a graph instead of a spanning tree. Since $e_{v^{\prime} v^{*}}+e_{v^{*} v}<3 e_{v^{\prime} v}$, the total length of $G$ satisfies $\overline{\|G\|}<3 \| \overline{\left\|E M T_{g}\right\|}$. Moreover, the relation 120 also holds for the graph $G$ when $i \in \mathcal{Y}$, i.e.,

$$
\frac{c_{0}(d)\left(n \gamma_{i}\left|\xi_{i}\right|\right)^{\frac{d-1}{d}}\left|\xi_{i}\right|^{\frac{1}{d}}}{\sum_{e \in d_{i}^{(G)}}|e|+c_{0}(d)\left(\left|V_{i}^{(G)}\right|\right)^{\frac{d-1}{d}}\left|\xi_{i}\right|^{\frac{1}{d}}} \leq 1,
$$

where $n \rightarrow \infty$, and $d_{i}^{(G)}, V_{i}^{(G)}$ are similar to those in 120 .

Moreover, for any $i \in \mathcal{X}$, let $\mathcal{J}_{i}$ denote the sum length of edge(s) of $G$ within $\xi_{i}$. It should be noted that if part of an edge is within $\xi_{i}$, only the part inside $\xi_{i}$ is considered. Since nodes in $\xi_{i}$ are uniformly distributed, $\mathcal{J}_{i}\left|\xi_{i}\right|^{-\frac{1}{d}}$ is greater than a constant with probability 1 . Thus, for any $i \in \mathcal{X}$,

$$
c_{0}(d)\left(n \gamma_{i}\left|\xi_{i}\right|\right)^{\frac{d-1}{d}}\left|\xi_{i}\right|^{\frac{1}{d}} \mathcal{J}_{i}^{-1} \leq \varpi^{*}
$$

where $\varpi^{*}=\max _{i \in \mathcal{X}}\left\{\frac{c_{0}(d)\left(n \gamma_{i}\left|\xi_{i}\right|\right)^{\frac{d-1}{d}}\left|\xi_{i}\right|^{\frac{1}{d}}}{\mathcal{J}_{i}}\right\}$ is a constant. Hence, according to (121), (122), the following relation holds

$$
\begin{aligned}
& \sum_{i \in \mathcal{X} \cup \mathcal{Y}} c_{0}(d)\left(n \gamma_{i}\left|\xi_{i}\right|\right)^{\frac{d-1}{d}}\left|\xi_{i}\right|^{\frac{1}{d}} \\
& \leq \sum_{i \in \mathcal{Y}} \sum_{e \in d_{i}^{(G)}}|e|+\varpi^{*} \sum_{i \in \mathcal{X}} \mathcal{J}_{i}+\sum_{i \in \mathcal{Y}} c_{0}(d)\left(\left|V_{i}^{(G)}\right|\right)^{\frac{d-1}{d}}\left|\xi_{i}\right|^{\frac{1}{d}} \\
& \leq \max \left\{3, \varpi^{*}\right\} \liminf _{n \rightarrow \infty} \overline{\left\|E M S T_{g}\right\|}+\sum_{i \in \mathcal{Y}} c_{0}(d)\left(\left|V_{i}^{(G)}\right|\right)^{\frac{d-1}{d}}\left|\xi_{i}\right|^{\frac{1}{d}},
\end{aligned}
$$

when $n \rightarrow \infty$. Furthermore, based on the edge replacement and the law of large numbers, for any $i \in \mathcal{Y}$,

$$
n \gamma_{i}\left|\xi_{i}\right|-\left|V_{i}^{(G)}\right| \geq \mid\left\{v: v \text { is within } \xi_{i}^{\prime}\right\}\left|>2^{-d}\left(1-\Delta_{0}\right) n \gamma_{i}\right| \xi_{i} \mid,
$$

with probability 1 when $n \rightarrow \infty$, where $0<\Delta_{0}<1$ is any constant. Hence, (123) can be further transformed as

$$
\liminf _{n \rightarrow \infty} \frac{\overline{\left\|E M S T_{g}\right\|}}{\sum_{i \in \mathcal{X} \cup \mathcal{Y}}\left(n \gamma_{i}\left|\xi_{i}\right|\right)^{\frac{d-1}{d}}\left|\xi_{i}\right|^{\frac{1}{d}}} \geq \mu c_{0}(d)
$$

where $\mu=\frac{\left(1-\left(1-2^{-d}\left(1-\Delta_{0}\right)\right)^{\frac{d-1}{d}}\right)}{\max \left\{3, \varpi^{*}\right\}}<1$ is a constant.

Consequently, based on (119) and (125), it is obvious that

$$
\lim _{n \rightarrow \infty} \frac{\overline{\left\|E M S T_{g}\right\|} n^{\frac{1-d}{d}}}{\int_{R^{d}} g_{n}(\mathbf{x})^{\frac{d-1}{d}} d \mathbf{x}}=\lim _{n \rightarrow \infty} \frac{\overline{\left\|E M S T_{g}\right\|} n^{\frac{1-d}{d}}}{\sum_{i \in \mathcal{X} \cup \mathcal{Y}}\left(\gamma_{i}\left|\xi_{i}\right|\right)^{\frac{d-1}{d}}\left|\xi_{i}\right|^{\frac{1}{d}}}=c(d),
$$

where $\mu c_{0}(d) \leq c(d) \leq \max \{2, \varpi\} c_{0}(d)$ is a constant. Thus, the Requirement 2 of Lemma 7 is satisfied. According to Lemma 7 Lemma 2 is proved.

\section{REFERENCES}

[1] P. Gupta, P. Kumar, "The capacity of wireless networks," in IEEE Transaction on Information Theory, vol. 46, no. 2, pp. 388-404, 2000.

[2] Z. Wang, H. Sadjadpour, J. Garcia-Luna-Aceves, "A Unifying Perspective on the Capacity of Wireless ad hoc Networks," in Proc. of IEEE INFOCOM 2008, Phoenix, AZ, USA, Apr. 2008.

[3] X. Li, "Multicast Capacity of Wireless Ad Hoc Networks," in IEEE/ACM Tracsactions on Networking, vol. 17, no. 3, Jun. 2009.

[4] S. Shakkottai, X. Liu and R. Srikant, "The Multicast Capacity of Large Multihop Wireless Networks," in IEEE/ACM Tracsactions on Networking, vol. 18, no. 6, Dec. 2010.

[5] P. Stuedi, O. Riva, G. Alonso, "ad hoc social networking using mand," in Proc. of ACM Mobicom 2008, San Francisco, California, USA, Sep. 2008.

[6] P. Fraigniaud, G. Giakkoupis, "On The Searchability of Small-World Networks with Arbitrary Underlying Structure," in Proc. of ACM STOC2010, Cambridge, USA, Jun. 2010.

[7] P. Fraigniaud, G. Giakkoupis, "The effect of power-law degrees on the navigability of small worlds," In Proc. 28th ACM Symposium on Principles of Distributed Computing, pp 240-249, 2009.

[8] Y. Ahn, S. Han, H. Kwak, S. Moon, H. Jeong, "Analysis of topological characteristics of huge online social networking services," in Proc. of $A C M W W W$, New York, NY, USA, pp. 835-844, 2007.

[9] B. Azimdoost, H. Sadjadpour, J. Garcia-Luna-Aceves, "The Impact of Social Groups on The Capacity of Wireless Networks," in Network Science Workshop, pp. 30-37, 2011.

[10] L. Ze, S. Haiying, W. Hailang, G. Liu, L. Jin, "SocialTube: P2P-assisted video sharing in online social networks," in Proc. of IEEE INFOCOM 2012, Orlando, FL, USA, Mar. 2012.

[11] M. Ilyas, M. Shafiq, A. Liu, H. Radha, "A distributed and privacy preserving algorithm for identifying information hubs in social networks," in Proc. of IEEE INFOCOM 2011, Shanghai, China, Apr. 2011.

[12] M. Taghizadeh, K. Micinski, S. Biswas, "Distributed Cooperative Caching In Social Wireless Networks," in IEEE Transactions on Mobile Computing, vol. PP, no. 99, 2012.

[13] S. Tang, J. Yuan, X. Mao, X. Li, W. Chen, G. Dai. "Relationship Classification in Large Scale Online Social Networks and Its Impact on Information Propagation", in Proc. of IEEE INFOCOM 2011, Shanghai, China, Apr. 2011.

[14] H. Inaltekin, C. Mung, H. Poor, "Average Message Delivery Time for Small-World Networks in the Continuum Limit," in IEEE Transactions on Mobile Computing, vol. 56, no. 9, pp. 4447-4470, 2010.

[15] J. Kleinberg, "The small-world phenomenon: an algorithm perspective," in 32nd Annual ACM Symposium on Theory of Computing, Portland, Oregon, USA, May 2000.

[16] N. Tichy, M. Tushman, C. Fombrun, "Social network analysis for organizations," in The Academy of Management Review, vol. 4, no. 4, pp. 507-519, 1979.

[17] Y. Ahn, S. Han, H. Kwak, S. Moon, H. Jeong, "Analysis of topological characteristics of huge online social networking services," in 16th international conference on World Wide Web, New York, USA, pp. 835-844, 2007. 
[18] R. Kumar, D. Liben-Nowell, et.al, "Theoretical Analysis of Geographic Routing in Social Networks," in MIT-CSAIL-TR-2005-040, Jun. 2005.

[19] D. Liben-Nowell, J. Novak, R. Kumar, P. Raghavan, A. Tomkins, R. Graham, "Geographic routing in social networks," in Proceedings of the National Academy of Sciences of the United States of America, vol. 102, no. 33, pp. 11623-11628, 2005.

[20] J. Ryu, W. Choi, "Bit Concatenation Based User Relaying in MIMO Broadcast Channels," in IEEE Transactions on Communications, vol. 60, no. 8, pp. 2208-2220, 2012.

[21] M. Steele, "Growth rates of euclidean minimal spanning trees with power weighted edges," in The Annals of Probability, vol. 16, no. 4, pp. 1767-1787, 1988

[22] T. Mitev, "New inequalities between elementary symmetric polynomials", in Journal of Inequalities in Pure and Applied Mathematics, vol. 4, no. 2, 2003.

[23] W. Frank Olver, Lozier, M. Daniel, Boisvert, F. Ronald, et al. NIST Handbook of Mathematical Functions, England :Cambridge, 2010.

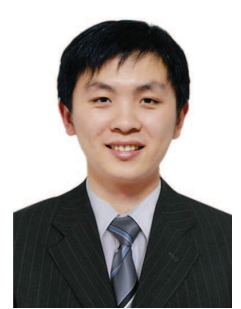

Yi Qin received the B.S. and M.S. degrees in electronic engineering from Shanghai Jiao Tong University, Shanghai, China, in 2009 and 2012, respectively. $\mathrm{He}$ is currently working toward the $\mathrm{Ph} . \mathrm{D}$. degree with Shanghai Jiao Tong University, Shanghai, China.

His research interests include D2D communication, D2D discovery, and Ad Hoc network scaling laws study.

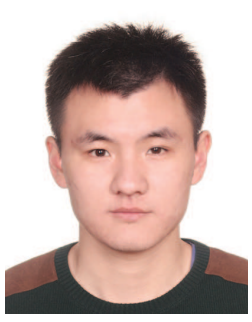

Riheng Jia received his B. E. degree in Electronics and Information Engineering from Huazhong University of Science and Technology, China, in 2012. $\mathrm{He}$ is currently pursuing the PHD degree in Electronic Engineering in Shanghai Jiao Tong University. His research of interests are in the area of wireless networks and information-centric networking.

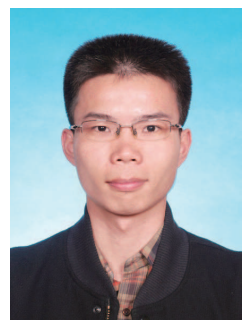

Jinbei Zhang received his B. E. degree in Electronic Engineering from Xidian University, Xi'an, China, in 2010, and is currently pursuing the Ph.D. degree in electronic engineering at Shanghai Jiao Tong University, Shanghai, China. His current research interests include network security, capacity scaling law and mobility models in wireless networks.

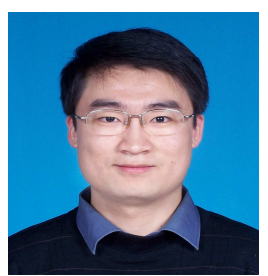

Weijie Wu is an Assistant Professor in School of Electronic, Information and Electrical Engineering, Shanghai Jiao Tong University. Before that, he was a research fellow working with Dr. Richard T.B. Ma in National University of Singapore, and a postdoctoral fellow working with Prof John C.S. Lui at The Chinese University of Hong Kong. He obtained his Ph.D. degree in computer science from The Chinese University of Hong Kong in August 2012, and Bachelor's degree in electronic \& information science and technology from Peking University in July 2008. When he was a Ph.D. student, he spent two months at National University of Singapore working as a research intern. His current research interests are in computer networks from mathematical modelling, data analytics, and economic perspectives. In particular, he is recently interested in network science (e.g., online social networks, large scale network with data implications, etc.), network economics (e.g, game theoretic analysis on communication networks, pricing and incentive design in network applications, etc.), and network optimization (e.g., resource allocation and pricing in cloud computing, information centric network, and P2P systems). His personal interests include table-tennis, badminton and hiking.

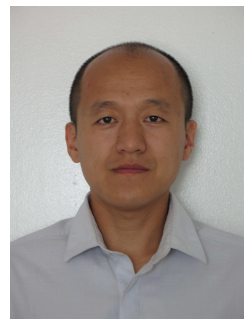

Xinbing Wang received the B.S. degree (with hons.) from the Department of Automation, Shanghai Jiaotong University, Shanghai, China, in 1998, and the M.S. degree from the Department of Computer Science and Technology, Tsinghua University, Beijing, China, in 2001. He received the Ph.D. degree, major in the Department of electrical and Computer Engineering, minor in the Department of Mathematics, North Carolina State University, Raleigh, in 2006. Currently, he is a professor in the Department of Electronic Engineering, Shanghai Jiaotong University, Shanghai, China. Dr. Wang has been an associate editor for IEEE/ACM Transactions on Networking and IEEE Transactions on Mobile Computing, and the member of the Technical Program Committees of several conferences including ACM MobiCom 2012, ACM MobiHoc 2012-2014, IEEE INFOCOM 2009-2014. 